\title{
STANDARD POLYNOMIALS IN MATRIX ALGEBRAS
}

\author{
BY
}

\author{
LOUIS H. ROWEN( $\left.{ }^{1}\right)$
}

ABSTRACT. Let $M_{n}(F)$ be an $n \times n$ matrix ring with entries in the field $F$, and let $S_{k}\left(X_{1}, \cdots, X_{k}\right)$ be the standard polynomial in $k$ variables. AmitsurLevitzki have shown that $S_{2 n}\left(X_{1}, \ldots, X_{2 n}\right)$ vanishes for all specializations of $X_{1}, \cdots, X_{2 n}$ to elements of $M_{n}(F)$. Now, with respect to the transpose, let $M_{n}^{-}(F)$ be the set of antisymmetric elements and let $M_{n}^{+}(F)$ be the set of symmetric elements. Kostant has shown using Lie group theory that for $\boldsymbol{n}$ even $S_{2 n-2}\left(X_{1}, \cdots, X_{2 n-2}\right)$ vanishes for all specializations of $X_{1}, \cdots, X_{2 n-2}$ to elements of $M_{n}^{-}(F)$. By strictly elementary methods we have obtained the following strengthening of Kostant's theorem:

$S_{2 n-2}\left(X_{1}, \cdots, X_{2 n-2}\right)$ vanishes for all specializations of $X_{1}, \cdots, X_{2 n-2}$ to elements of $M_{n}^{-}(F)$, for all $n$.

$S_{2 n-1}\left(X_{1}, \cdots, X_{2 n-1}\right)$ vanishes for all specializations of $X_{1}, \cdots, X_{2 n-2}$ to elements of $M_{n}^{-}(F)$ and of $X_{2 n-1}$ to an element of $M_{n}^{+}(F)$, for all $n$.

$S_{2 n-2}\left(X_{1}, \cdots, X_{2 n-2}\right)$ vanishes for all specializations of $X_{1}, \cdots, X_{2 n-3}$ to elements of $M_{n}^{-}(F)$ and of $X_{2 n-2}$ to an element of $M_{n}^{+}(F)$, for $n$ odd.

These are the best possible results if $F$ has characteristic 0 ; a complete analysis of the problem is also given if $F$ has characteristic 2.

Introduction. The object of this paper is to prove the results described in the abstract. The method of proof is to exploit certain properties of the trace (given in $\$ 1$ ) in connection with an undirected graph whose edges correspond to elementary symmetric and antisymmetric matrices (see $\$ 2)$. $\$ \S_{3}-6$ consist of manipulations of the graph to prove the main theorem (Theorem 1). Although Theorem 1 is sharp in characteristic 0 (as shown via counterexamples in $\$ 8$ ), more results can be obtained in characteristic 2 and, at times, in odd characteristic (viz. \$7). The sharpness of these results is also explained in $\$ 8$. In $\$ 9$ the relationship of Theorem 1 and the theory of identities of rings with involution is given.

In recent months two other people, Joan Hutchinson and Frank Owens, inde-

Presented to the Society, January 25, 1973 under the title Standard identities for matrix rings with involution; received by the editors March 23, 1973.

AMS (MOS) subject classifications (1970). Primary 15A24, 15A57, 16A28, 16A38; Secondary 05C30, $05 C 35$.

Key words and phrases. Antisymmetric, involution, matrix algebra, polynomial identity, standard identity, symmetric, transpose.

(1) The author is a doctoral candidate at Yale University working under a NSF research grant, and would like to thank N. Jacobson, his advisor, and N. White, both of whom offered invaluable advice in the preparation of this paper. 
pendently studying similar questions, have announced elementary proofs of Kostant's theorem and its extension to $n$ odd, which is $\mathfrak{L}_{0}(n, 2 n-2,0)$ in the terminology of this paper. I have not yet had the opportunity to see their proofs in detail, although Mr. Owens has issued a summary of his proof.

1. Preliminary results. Define the standard polynomial $S_{k}$ as follows: $s_{k}\left(X_{1}, X_{2}, \cdots, X_{k}\right)=\Sigma_{\pi}(s g \pi) X_{\pi 1} X_{\pi 2} \cdots X_{\pi k}$, taking the sum over all permutations $\pi$ of $(1, \ldots, k)$ where $s g \pi$ is the sign of $\pi,+1$ if $\pi$ is even and -1 if $\pi$ is odd. Clearly $S_{k}$ is multilinear, i.e. linear in each of its variables, and alternating.

An immediate consequence of the definition is

$$
\begin{aligned}
s_{k+1}\left(X_{1}, \cdots, X_{k+1}\right)= & X_{1} s_{k}\left(X_{2}, \ldots, X_{k+1}\right)-X_{2} s_{k}\left(X_{1}, X_{3}, \ldots, X_{k+1}\right) \\
& +\cdots+(-1)^{k} X_{k+1} s_{k}\left(X_{1}, \ldots, X_{k}\right) .
\end{aligned}
$$

Let $\Omega$ be a domain and let $M_{n}(\Omega)$ be the algebra of $n \times n$ matrices with entries in $\Omega$. There is a canonical base $B_{n}$ of matric units $e_{i j}, 1 \leq i, j \leq n$, where $e_{i j}$ is the matrix whose only nonzero entry is 1 in the $(i, j)$-position. Clearly $\sum_{i=1}^{n} e_{i i}=1$, and multiplication in $M_{n}(\Omega)$ is induced by $e_{i j} e_{r s}=\delta_{j r} e_{i s}$. Let $B_{n}^{+}=$ $\left\{e_{i i} \mid 1 \leq i \leq n\right\} \cup\left\{e_{i j}+e_{j i} \mid 1 \leq i<j \leq n\right\}$ and let $B_{n}^{-}=\left\{e_{i j}-e_{j i} \mid 1 \leq i<j \leq n\right\}$. $B_{n}^{+}$and $B_{n}^{-}$are each sets of elements of $M_{n}(\Omega)$ which are linearly independent over $\Omega$. Let $M_{n}^{+}(\Omega)$ and $M_{n}^{-}(\Omega)$ be the submodules of $M_{n}(\Omega)$ for which $B_{n}^{+}$and $B_{n}^{-}$are the respective bases. If char $\Omega \neq 2$ then $M_{n}^{+}(\Omega)$ and $M_{n}^{-}(\Omega)$ are the sets of symmetric and antisymmetric elements of $\Omega$, with respect to the transpose $(*)$ given by its action on the base, $e_{i j}^{*}=e_{j i}$.

Consider now the class of sentences $\left\{\mathfrak{L}_{p}(n, k, t)\right\}$, where $\mathfrak{L}_{p}(n, k, t)$ says, "For any field $F$ of characteristic $p, S_{k}\left(A_{1}, \cdots, A_{k}\right)=0$ for all sets of matrices $\left\{A_{1}, \cdots, A_{k}\right\}$ with $t$ elements in $M_{n}^{+}(F)$ and with $(k-t)$ elements in $M_{n}^{-}(F)$." Let $\pi$ be any permutation of $(1, \cdots k)$. Then from the definition of $S_{k}$, we see that $S_{k}\left(A_{1}, \cdots, A_{k}\right)=0 \Leftrightarrow S_{k}\left(A_{\pi 1}, \cdots, A_{\pi k}\right)=0$. Thus, an equivalent formulation of $\mathcal{L}_{p}(n, k, t)$ says, "For any field $F$ of characteristic $p, S_{k}\left(A_{1}, \cdots, A_{k}\right)=0$ for any $A_{1}, \cdots, A_{k-t}$ in $M_{n}^{-}(F), A_{k-t+1}, \cdots, A_{k}$ in $M_{n}^{+}(F)$." Let us say by convention that $\mathscr{L}_{p}(n, k, t)$ holds if $k \leq 0$ or if $t \leq-1$ or if $t>k$.

Lemma 1. $\mathfrak{L}_{p}(n, k, t)$ is equivalent to the sentence, "In characteristic $p$, $S_{k}\left(A_{1}, \cdots, A_{k}\right)=0$ for all $A_{1}, \cdots, A_{k-t}$ in $B_{n}^{-}$and $A_{k-t+1}, \cdots, A_{k}$ in $B_{n}^{+}, "$ Furthermore we have the implications:

(a) $\mathfrak{£}_{0}(n, k, t)$ implies $\mathfrak{\complement}_{p}(n, k, t)$ for all $p$.

(b) $\mathfrak{L}_{p}(n, k, t)$ and $\mathscr{L}_{p}(n, k, t-1)$ imply $\mathfrak{L}_{p}(n, k+1, t)$.

(c) $\mathfrak{L}_{p}(n, k, t)$ implies $\mathfrak{\complement}_{p}(n-1, k, t)$.

Proof. These assertions are all trivial. Since $S_{k}$ is multilinear, it suffices 
to consider only matrices in $B_{n}^{-}$and $B_{n}^{+}$. Consequently, (a) is immediate, (b) follows from (1), and (c) is a result of the natural embedding $B_{n} \hookrightarrow B_{n+1}$ Q.E.D.

Remark. In view of Lemma 1 , one can demonstrate $\mathfrak{L}_{p}(n, k, t)$ by showing there exists a domain $\Omega$ of characteristic $p$ such that $S_{k}\left(A_{1}, \cdots, A_{k}\right)=0$ for all $A_{1}, \cdots, A_{k-t}$ in $M_{n}^{-}(\Omega)$ and $A_{k-t+1}, \cdots, A_{k}$ in $M_{n}^{+}(\Omega)$. When $p=0$ we will at times take $\Omega=Z$, the integers, $\Omega=Q$, the rational numbers, or $\Omega=R$, the real numbers.

A major result concerning the polynomial $S_{k}$ is

Theorem (Amitsur and Levitzki $[1]) . S_{2 n}\left(A_{1}, \cdots, A_{2 n}\right)=0$ for all $A_{1}, \cdots, A_{2 n}$ in $M_{n}(F), F$ any field.

Clearly the Amitsur-Levitzki theorem implies $\mathcal{L}_{p}(n, 2 n, t)$ for all $p$, all $t$. Conversely, we claim that the Amitsur-Levitzki theorem is implied by $\mathscr{L}_{0}(n, 2 n, t)$ for all $t$. Indeed, it is enough to verify the Amitsur-Levitzki theorem for elements of $B_{n}$, since $S_{2 n}$ is multilinear; hence we may assume $F$ has characteristic 0 . But in this case $B_{n}^{+} \cup B_{n}^{-}$is a base for $M_{n}(F)$, so it is enough to show that $S_{2 n}\left(A_{1}, \cdots, A_{2 n}\right)=0$ for $A_{1}, \cdots, A_{2 n}$ in $B_{n}^{+} \cup B_{n}^{-}$. Reordering these matrices, we may assume $A_{1}, \cdots, A_{2 n-t} \in B_{n}^{-}$and $A_{2 n-t+1}, \cdots, A_{2 n} \in B_{n}^{+}$, for some $t$. But then $\mathscr{L}_{0}(n, 2 n, t)$ implies $S_{2 n}\left(A_{1}, \cdots, A_{2 n}\right)=0$, so the claim is established.

Considering the Amitsur-Levitzki theorem as a consequence of $\mathfrak{L}_{0}(n, 2 n, t)$ for all $t$, one may wonder which other sentences are true. Lemma 1 shows that $\mathcal{L}_{p}(n, k, t)$ is true for $k \geq 2 n$. In a brilliant paper linking the theory of standard polynomials to Lie group theory, B. Kostant [3, p. 247] proved, among other things,

Theorem (Kostant). $\mathscr{\complement}_{0}(n, 2 n-2,0)$ for $n$ even.

The main objective of this paper is to give the following complete characterization of the sentences $\mathfrak{L}_{0}$ in the following result, proved by elementary methods; an elementary proof of Kostant's theorem will be a by-product.

Theorem 1. $\mathfrak{Q}_{0}(n, 2 n-1, t)$ for $t=0$ or $t=1, \mathfrak{Q}_{0}(n, 2 n-2,0)$ for all $n$. If $n$ is odd, $\mathfrak{\complement}_{0}(n, 2 n-2,1)$.

Counterexamples are given for all sentences $\mathfrak{L}_{0}(n, k, t)$, all situations not already discussed, namely for $2 \leq t \leq k<2 n, 0 \leq t \leq k \leq 2 n-3$, all $n$, or $t=1$, $k=2 n-2, n$ even.

A complete analysis of the characteristic 2 case is also given in the much easier

Theorem 2. $\mathfrak{L}_{2}(n, k, t)$ for $k \geq n+t$.

Counterexamples are given for all sentences $\mathfrak{Q}_{p}(n, k, t), k<n+t$. 
Before starting the proof of Theorem 1, which is largely graph-theoretic, we give some easy algebraic results.

Lemma $2 . \mathscr{L}_{p}(n, k, t)$ implies $\mathfrak{L}_{p}(n-1, k-1, t-1)$ and $\mathfrak{L}_{p}(n-1, k-1, t)$, for any $p$.

Proof. Assume $\mathscr{L}_{p}(n, k, t)$. To show $\mathfrak{L}_{p}(n-1, k-1, t-1)$, we must show that for all $A_{1}, \cdots, A_{k-t}$ in $M_{n-1}^{-}(F)$ and all $A_{k-i+1}, \cdots, A_{k-1}$ in $M_{n-1}^{+}(F)$, $S_{k-1}\left(A_{1}, \cdots, A_{k-1}\right)=0$. Well suppose $S_{k-1}\left(A_{1}, \cdots, A_{k-1}\right)=\sum_{i, j=1}^{n-1} a_{i j} e_{i j} \cdot$ Embedding $M_{n-1}(F)$ canonically into $M_{n}(F)$, let $A_{k}=e_{v n}+e_{n v}, v \in\{1, \ldots, n-1\}$. $A_{k} \in M_{n}^{+}(F)$, so by hypothesis $S_{k}\left(A_{1}, \cdots, A_{k}\right)=0$. On the other hand, if $1 \leq r, s \leq$ $k-1$, then $A_{r}\left(e_{v n}+e_{n v}\right) A_{s}=0$, so

$$
\begin{aligned}
S_{k}\left(A_{1}, \ldots, A_{k}\right) & =S_{k-1}\left(A_{1}, \ldots, A_{k-1}\right) A_{k}+(-1)^{k-1} A_{k} S_{k-1}\left(A_{1}, \ldots, A_{k-1}\right) \\
& =\left(\sum_{i, j=1}^{n-1} a_{i j} e_{i j}\right)\left(e_{v n}+e_{n v}\right)+(-1)^{k-1}\left(e_{v n}+e_{n v}\right)\left(\sum_{i, j=1}^{n-1} a_{i j} e_{i j}\right) \\
& =\sum_{i=1}^{n-1} a_{i v} e_{i n}+(-1)^{k-1} \sum_{j=1}^{n} a_{v j} e_{n j} \cdot
\end{aligned}
$$

Thus, $0=\alpha_{u v}$ for all $u, v$ in $\{1, \cdots, n-1\}$, implying $S_{k-1}\left(A_{1}, \cdots, A_{k-1}\right)=0$, as was to be shown. Hence, $\mathscr{L}_{p}(n, k, t)$ implies $\mathscr{L}_{p}(n-1, k-1, t-1)$.

The proof $\mathscr{L}_{p}(n, k, t)$ implies $\mathscr{\complement}_{p}(n-1, k-1, t)$ is analogous if we let $A_{k}=$ $e_{\nu n}-e_{n v}$ at the corresponding place. Q.E.D.

It is well known that the trace defines a symmetric bilinear form on $M_{n}(F)$, given by $\left(A_{1}, A_{2}\right)=\operatorname{tr}\left(A_{1} A_{2}\right)$ for $A_{1}, A_{2}$ in $M_{n}(F)$. Moreover, if $A_{1}=\Sigma a_{i j} e_{i j}$ then $\left(A_{1}, e_{u v}\right)=a_{v u}$, which shows that the trace bilinear form is nondegenerate. The following lemmas are based on ideas used by Kostant [3].

Lemma 3. If char $F \neq 2$, then $M_{n}^{+}(F)$ and $M_{n}^{-}(F)$ are nondegenerate subspaces of $M_{n}(F)$, relative to the trace bilinear form.

Proof. Since char $F \neq 2, M_{n}(F)=M_{n}^{+}(F) \oplus M_{n}^{-}(F)$, given by $A=1 / 2\left(A+A^{*}\right)+$ $1 / 2\left(A-A^{*}\right)$. The lemma will follow from the fact that $M_{n}^{+}(F)$ and $M_{n}^{-}(F)$ are orthogonal under the trace bilinear form. Indeed, if $Y \in M_{n}^{+}(F)$ and $K \in M_{n}^{-}(F)$, then $(Y, K)=\operatorname{tr} Y K=\operatorname{tr} K Y=\operatorname{tr}(K Y)^{*}=\operatorname{tr} Y^{*} K^{*}=\operatorname{tr}(-Y K)=-(Y, K)$, so $(Y, K)=$ O. Q.E.D.

Lemma 4. Let $A_{1}, \cdots, A_{k-t} \in M_{n}^{-}(F), A_{k-t+1}, \cdots, A_{k} \in M_{n}^{+}(F)$. If $t$ is even then the matrix $S_{k}\left(A_{1}, \cdots, A_{k}\right)$ is symmetric if $k \equiv 0$ or $3(\bmod 4)$, and ant isymmetric if $k \equiv 1$ or $2(\bmod 4)$. If $t$ is odd then the matrix $S_{k}\left(A_{1}, \cdots, A_{k}\right)$ is antisymmetric if $k \equiv 0$ or $3(\bmod 4)$, and symmetric if $k \equiv 1$ or $2(\bmod 4)$. 


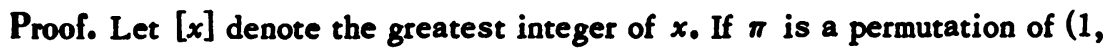

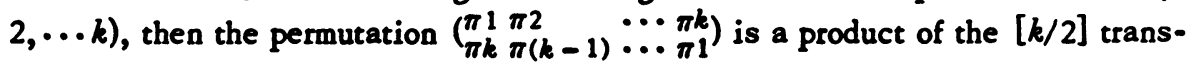
positions $(\pi 1, \pi k),(\pi 2, \pi(k-1)), \cdots,(\pi[k / 2], \pi(k-[k / 2]))$. Thus, $s_{k}\left(A_{1}, A_{2}, \cdots, A_{k}\right)=$ $(-1)^{[k / 2]} S_{k}\left(A_{k}, A_{k-1}, \cdots, A_{1}\right)$. Now $\left.\left(A_{\pi 1} A_{\pi 2} \cdots A_{\pi k}\right)^{*}=(-1)^{k-t} A_{\pi k} A_{\pi(k-1}\right)^{\cdots A_{\pi 1}}$ Thus,

$$
\begin{gathered}
S_{k}\left(A_{1}, A_{2}, \ldots, A_{k}\right)^{*}=(-1)^{k-t} S_{k}\left(A_{k}, A_{k-1}, \ldots, A_{1}\right) \\
=(-1)^{k-t+[k / 2]} S_{k}\left(A_{1}, A_{2}, \ldots, A_{k}\right) .
\end{gathered}
$$

The sign $(-1)^{k-t+[k / 2]}$ gives the desired results. Q.E.D.

Lemma 5 (Kostant [3, p. 244]). For $A_{i}$ in $M_{n}(F), 1 \leq i \leq 2 k-1$,

$$
\text { tr } S_{2 k-1}\left(A_{1}, \cdots, A_{2 k-1}\right)=(2 k-1) \operatorname{tr}\left(S_{2 k-2}\left(A_{1}, \ldots, A_{2 k-2}\right) A_{2 k-1}\right) \text {. }
$$

Proof. For any permutation $\pi$ of $(1,2, \ldots, 2 k-2)$ and for $1 \leq j \leq 2 k-3$,

Therefore,

$$
\begin{aligned}
\operatorname{tr}\left(A_{\pi(j+1)} A_{\pi(j+2)} \cdots A_{\pi(2 k-2)} A_{2 k-1} A_{\pi 1} A_{\pi 2} \cdots A_{\pi j}\right) \\
\quad=\operatorname{tr}\left(A_{\pi 1} \cdots A_{\pi j} A_{\pi(j+1)} \cdots A_{\pi(2 k-2)} A_{2 k-1}\right) .
\end{aligned}
$$

$$
\begin{gathered}
\operatorname{tr}\left(A_{\pi 1} A_{\pi 2} \cdots A_{\pi(2 k-2)} A_{2 k-1}+\sum_{j=1}^{2 k-3} A_{\pi(j+1)} A_{\pi(j+2)} \cdots A_{\pi(2 k-2)} A_{2 k-1} A_{\pi 1} \cdots\right. \\
\left.A_{\pi j}+A_{2 k-1} A_{\pi 1} \cdots A_{\pi(2 k-2)}\right) \\
=(2 k-1) \operatorname{tr}\left(A_{\pi 1} A_{\pi 2} \cdots A_{\pi(2 k-2)} A_{2 k-1}\right) .
\end{gathered}
$$

Moreover, $s_{8} \pi$ is the sign of the permutation

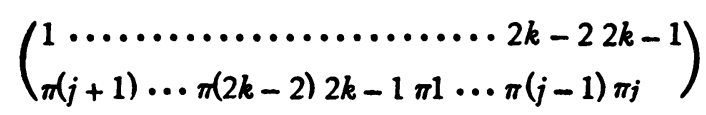

because the permutation

$$
\left(\begin{array}{l}
\pi 1 \ldots \ldots \ldots \ldots \ldots \ldots \ldots \ldots \ldots(2 k-2) 2 k-1 \\
\pi(j+1) \ldots \ldots(2 k-2) 2 k-1 \pi 1 \cdots \pi(j-1) \pi j
\end{array}\right)
$$

is always even (its sign is $(-1)^{(2 k-1-j) j}=+1$ for any $\left.k, j\right)$. Hence, summing over all $\pi$, we see tr $S_{2 k-1}\left(A_{1}, \cdots, A_{2 k-1}\right)=(2 k-1) \operatorname{tr}\left(S_{2 k-2}\left(A_{1}, \cdots, A_{2 k-2}\right) A_{2 k-1}\right)$. Q.E.D.

Putting together the above lemmas yields the following key result

Lemma 6. (a) Let $k$ be an even integer, and let $F$ be a field in wbicb $2(2 k-1)$ bas an inverse. If tr $S_{2 k-1}\left(A_{1}, \cdots, A_{2 k-1}\right)=0$ for all $A_{1}, \cdots, A_{2 k-1}$ in $M_{n}^{-}(F)$, then $S_{2 k-2}\left(A_{1}, \cdots, A_{2 k-2}\right)=0$ for all $A_{1}, \cdots, A_{2 k-2}$ in $M_{n}^{-}(F)$. 
(b) If $n$ is odd and if $\operatorname{tr}\left(S_{2 n-1}\left(A_{1}, \cdots, A_{2 n-1}\right)\right)=0$ for all $A_{1}, \cdots, A_{2 n-2}$ in $M_{n}^{-}(Q), A_{2 n-1}$ in $M_{n}^{+}(Q)$, then $\mathscr{\varrho}_{0}(n, 2 n-2,0), \mathscr{\complement}_{0}(n, 2 n-2,1)$,

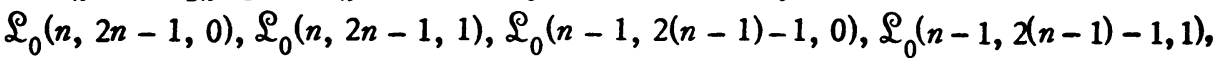
$\mathcal{L}_{0}(n-1,2(n-1)-2,0)$.

Proof. (a) By Lemma $5, \operatorname{tr}\left(S_{2 k-2}\left(A_{1}, \cdots, A_{2 k-2}\right) A_{2 k-1}\right)=0$ for all $A_{1}, \cdots$, $A_{2 k-1}$ in $M_{n}^{-}(F)$. But $S_{2 k-2}\left(A_{1}, \cdots, A_{2 k-2}\right) \in M_{n}^{-}(F)$ by Lemma 4 . Hence by the nondegeneracy of the trace bilinear form on $M_{n}^{-}(F), S_{2 k-2}\left(A_{1}, \cdots, A_{2 k-2}\right)=0$.

(b) By Lemma $5, \operatorname{tr}\left(S_{2 n-2}\left(A_{1}, \cdots, A_{2 n-2}\right) A_{2 n-1}\right)=0$ for all $A_{1}, \cdots, A_{2 n-2}$ in $M_{n}^{-}(\mathbf{Q}), A_{2 n-1}$ in $M_{n}^{+}(\mathbf{Q})$. But $S_{2 n-2}\left(A_{1}, \cdots, A_{2 n-2}\right)$ is symmetric by Lemma

4. By the nondegeneracy of the trace bilinear form on $M_{n}^{+}(Q), S_{2 n-2}\left(A_{1}, \cdots, A_{2 n-2}\right)=$ 0 , implying $\varrho_{0}(n, 2 n-2,0)$. Likewise, since

$$
S_{2 n-1}\left(A_{1}, A_{2}, \cdots, A_{2 n-1}\right)=S_{2 n-1}\left(A_{2}, \ldots, A_{2 n-1}, A_{1}\right),
$$

the hypothesis implies $\operatorname{tr}\left(S_{2 n-2}\left(A_{2}, \cdots, A_{2 n-1}\right) A_{1}\right)=0$, all $A_{1}, \cdots, A_{2 n-2}$ in $M_{n}^{-}(Q)$ and $A_{2 n-1}$ in $M_{n}^{+}(Q)$. Since $S_{2 n-2}\left(A_{2}, \cdots, A_{2 n-1}\right)$ is antisymmetric by Lemma 4, we conclude $S_{2 n-2}\left(A_{2}, \cdots, A_{2 n-1}\right)=0$, proving $\mathscr{\complement}_{0}(n, 2 n-2,1)$. From $\mathscr{L}_{0}(n, 2 n-2,0)$ and $\mathscr{L}_{0}(n, 2 n-2,1)$ we get $\mathscr{L}_{0}(n, 2 n-1,0)$ and $\mathscr{L}_{0}(n, 2 n-1,1)$ by Lemma 1 , and from $\mathfrak{L}_{0}(n, 2 n-2,1)$ we get $\mathfrak{L}_{0}(n-1,2(n-1)-1,1)$ and $\mathfrak{L}_{0}(n-1,2(n-1)-1,0)$ by Lemma 2 . But then we get $\mathfrak{L}_{0}(n-1,2(n-1)-2,0)$ by part (a). Q.E.D.

In this paper, all monomials will be assumed to have degree $\leq 1$ in each indeterminate.

Often we shall be interested in the sums of certain monomials of the polynomial $S_{k}$. In particular, we formulate

Definition. Let $v_{1}, v_{2}, \cdots, v_{m}$ be monomials in $X_{1}, \cdots, X_{k}$ such that the degree of $X_{i}$ in $v_{1} \ldots v_{m}$ is 0 or 1 , all $j$. Then $s_{k}\left(X_{1}, \cdots, X_{k} ; v_{1}, \ldots, v_{m}\right)$ is defined as the sum of exactly those (signed) submonomials of $s_{k}\left(X_{1}, \ldots, X_{k}\right)$ in which $V_{1}, \cdots, V_{m}$ are each submonomials. (In other words, a typical monomial of $S_{k}\left(X_{1}, \cdots, X_{k} ; V_{1}, \cdots, V_{m}\right)$ has the form

$$
\pm T_{0} V_{\mu 1} T_{1} V_{\mu 2} T_{2} \cdots T_{m-1} V_{\mu m} T_{m}
$$

where $T_{0}, \cdots, T_{m}$ are arbitrary monomials in $X_{1}, \cdots, X_{k}$ and $\mu$ is a permutation of $(1, \cdots, m)$.

Lemma 7. Let $A_{1}, \cdots, A_{k} \in B_{n}^{+} \cup B_{n}^{-}$, and for $r<(k-1) / 2$ let $\tilde{A}=$ $A_{1} A_{2} \cdots A_{2 r+1}+(-1)^{r} A_{2 r+1} A_{2 r} \cdots A_{1}$. If s elements of $\left\{A_{1}, \cdots, A_{2 r+1}\right\}$ are symmetric and if the other $(2 r+1-s)$ elements are antisymmetric then 
$\tilde{A} \in B_{n}^{-}$when $s+r$ is even, and $\tilde{A} \in B_{n}^{+}$when $s+r$ is odd. Moreover, for any $m \geq 0$,

$$
\begin{aligned}
S_{k}\left(A_{1}, \cdots, A_{k} ; A_{1} A_{2} \cdots A_{2 r+1}, V_{1}, \cdots, V_{m}\right) \\
+S_{k}\left(A_{1}, \cdots, A_{k} ; A_{2 r+1} A_{2 r} \cdots A_{1}, V_{1}, \cdots, V_{m}\right) \\
=S_{k-2 r}\left(\tilde{A}, A_{2 r+2}, \cdots, A_{k} ; V_{1}, \cdots, V_{m}\right)
\end{aligned}
$$

Proof.

$$
\begin{aligned}
\tilde{A}^{*} & =A_{2 r+1}^{*} \cdots A_{2}^{*} A_{1}^{*}+(-1)^{r} A_{1}^{*} \cdots A_{2 r}^{*} A_{2 r+1}^{*} \\
& =(-1)^{2 r+1-s}\left(A_{2 r+1} \cdots A_{2} A_{1}+(-1)^{r} A_{1} \cdots A_{2 r} A_{2 r+1}\right) \\
& =(-1)^{r+1-s} \tilde{A} .
\end{aligned}
$$

Thus $\tilde{A}$ is symmetric or antisymmetric depending on whether $r+s$ is odd or even. The rest of the lemma is immediate. Q.E.D.

2. Beginning of proof of Theorem 1: The graph $\Gamma$. In view of Lemma 6 , Theorem 1 is implied by the statement, " $\varrho_{0}(n, 2 n-1,1)$ for all odd $n>1$." Therefore, to prove Theorem 1 it suffices to show, for all $n>1, \mathfrak{L}_{0}(n, 2 n-1,1)$ and $\mathfrak{L}_{0}(n, 2 n-1,0)$. This assertion will be proved by induction on $n$. Clearly, $\mathscr{L}_{0}(n, 2 n-1,1)$ and $\mathscr{L}_{0}(n, 2 n-1,0)$ for $n=2$, so we shall assume $\mathscr{L}_{0}(m, 2 m-1,1)$ and $\mathscr{L}_{0}(m, 2 m-1,0)$ for all $m, 2 \leq m<n$, and will show $\mathscr{L}_{0}(n, 2 n-1,1)$ and $\mathfrak{L}_{0}(n, 2 n-1,0)$.

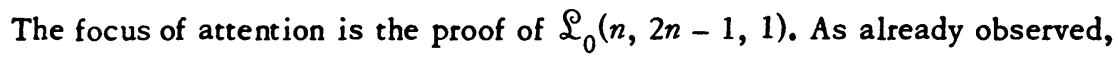
we need only show $S_{2 n-1}\left(A_{1}, \cdots, A_{2 n-1}\right)=0$ for all $A_{1}, \cdots, A_{2 n-2}$ in $M_{n}^{-}(\mathbf{R})$, $A_{2 n-1}$ in $M_{n}^{+}(\mathbf{R})$, and in fact if $n$ is odd we need only $\operatorname{tr} S_{2 n-1}\left(A_{1}, \cdots, A_{2 n-1}\right)=$ 0 , by Lemma 6 . Since $S_{2 n-1}$ is multilinear we may assume $A_{2 n-1} \in B_{n}^{+}$. We claim that it suffices to consider $A_{2 n-1}$ in $\left\{e_{i i} \mid 1 \leq i \leq n\right\}$. Indeed, identifying 1 with the multiplicative unit of $M_{n}(\mathrm{R})$, let us assume $A_{2 n-1}=e_{i j}+e_{j i}$ and let $Y=1+$ $(\sqrt{ } 2 / 2-1)\left(e_{i i}+e_{j j}\right)+(\sqrt{ } 2 / 2)\left(e_{i j}-e_{j i}\right) \cdot Y^{*} Y=Y Y^{*}=1$ and $Y A_{2 n-1} Y^{*}=e_{i i}-$ $e_{j j}$, so

$$
\begin{aligned}
& Y S_{2 n-1}\left(A_{1}, \ldots, A_{2 n-1}\right) Y^{*}=S_{2 n-1}\left(Y A_{1} Y^{*}, \ldots, Y A_{2 n-2} Y^{*}, e_{i i}-e_{j j}\right) \\
& \quad=S_{2 n-1}\left(Y A_{1} Y^{*}, \ldots, Y A_{2 n-2} Y^{*}, e_{i i}\right)-S_{2 n-1}\left(Y A_{1} Y^{*}, \ldots, Y A_{2 n-2} Y^{*}, e_{j j}\right)
\end{aligned}
$$

Moreover, $Y \operatorname{tr} S_{2 n-1}\left(A_{1}, \cdots, A_{2 n-1}\right) Y^{*}=\operatorname{tr}\left(Y S_{2 n-1}\left(A_{1}, \cdots, A_{2 n-1}\right) Y^{*}\right)=$ tr $S_{2 n-1}\left(Y A_{1} Y^{*}, \ldots, Y A_{2 n-2} Y^{*}, e_{i i}\right)-\operatorname{tr} S_{2 n-1}\left(Y A_{1} Y^{*}, \ldots, Y A_{2 n-2} Y^{*}, e_{j j}\right)$. Since $Y A_{r} Y^{*}$ is antisymmetric, $1 \leq r \leq 2 n-2$, the claim is established. 
Thus we may assume $A_{2 n-1} \in\left\{e_{i i} \mid 1 \leq i \leq n\right\}$, and by symmetry we shall assume $A_{2 n-1}=e_{11}$. Since $S_{2 n-1}$ is multilinear and alternating, we may assume $A_{1}, \cdots, A_{2 n-2} \in B_{n}^{-}$, and $A_{i} \neq A_{j}$ if $i \neq j$.

Generalizing the situation slightly, suppose $u>1$ is arbitrary and $\delta$ is a subset of $B_{u}^{-} \cup\left\{e_{11}\right\}$. There is a graph $\Gamma(\mathcal{S})$ associated with $\delta$, constructed as follows:

The vertices of $\Gamma(\mathcal{S})$ are the indices $1, \ldots, u$, and each element $Y$ of $\delta$ is represented by an edge $\hat{Y}$ of $\Gamma(\delta)$, where $\hat{Y}=\{1,1\}$ if $Y=e_{11}$ and $\hat{Y}=\{i, j\}$ if $Y=e_{i j}-e_{j i}$. Using the terminology of [4], we view $\Gamma(\delta)$ as an undirected graph, with at most one edge joining any two given vertices. $\Gamma(\mathcal{S})$ has a loop, $\{1,1\}$, if and only if $e_{11} \in \mathrm{S}$.

Let $\mathcal{S}=\left\{Y_{1}, \cdots, Y_{v}\right\}, v \geq 2$. Let $\left\langle\hat{Y}_{\pi 1}, \cdots, \hat{Y}_{\pi k}\right\rangle$ be a sequence of edges, $\pi$ a permutation of $(1, \ldots, v)$ such that for $1 \leq r \leq k$ one may order the endpoints $i_{r}$ and $j_{r}$ of $\hat{Y}_{\pi r}$ in a way so that $i_{2}=j_{1}, i_{3}=j_{2}, \cdots, i_{k}=j_{k-1}$. Then we call $\left\{\left\langle\hat{Y}_{\pi 1}, \cdots, \hat{Y}_{\pi k}\right\rangle, i_{1}, j_{k}\right\}$ a path of length $k$ with initial vertex $i_{1}$ and terminal vertex $j_{k}$ (cf. [4, p. 22]). The vertices $i_{1}$ and $j_{k}$ will be called end vertices, whereas $j_{1}, i_{k}$ and $i_{r}$ and $j_{r}, 2 \leq r \leq k-1$, will be called intermediate vertices. Since a given vertex may be incident to many edges, an index might be both an intermediate and an end vertex of the same path. By definition, the intermediate vertices of a path occur in pairs.

Lemma 8. Let $k \geq 2$. Given a sequence of edges $\left\langle\hat{Y}_{\pi 1}, \cdots, \hat{Y}_{\pi k}\right\rangle$ of $\Gamma(\mathcal{S})$, there is at most one value each of $i_{1}$ and $j_{k}$ sucb that $\left\{\left\langle\hat{Y}_{\pi 1}, \ldots, \hat{Y}_{\pi k}\right\rangle, i_{1}, j_{k}\right\}$ is a path. Moreover there is a $1: 1$ correspondence between paths of $\Gamma(\delta)$ of lengtb $k$ and nonzero monomials in $Y_{1}, \cdots, Y_{v}$ of degree $k_{.}$In this correspondence, the monomial $Y_{\pi 1} \ldots Y_{\pi k}$ associated with the path $\left\{\left\langle\hat{Y}_{\pi 1}, \ldots, \hat{Y}_{\pi k}\right\rangle, i_{1}, j_{k}\right\}$ bas value $\pm e_{i_{1} j_{k}} \cdot$

Proof. First assume $k=2$. If $\left\langle\hat{Y}_{\pi 1}, \hat{Y}_{\pi 2}\right\rangle$ is the sequence of edges of a path, then we must be able to write $\hat{Y}_{\pi 1}, \hat{Y}_{\pi 2}$ respectively as $\left\{i_{1}, j_{1}\right\},\left\{i_{2}, j_{2}\right\}$, where $j_{1}=i_{2}$. Since $\hat{Y}_{\pi 1} \neq \hat{Y}_{\pi 2}$, we have $i_{1} \neq j_{2}$, so the first assertion of the lemma is immediate for $k=2$.

For $k>2$, if $\left\langle\hat{Y}_{\pi 1}, \cdots, \hat{Y}_{\pi k}\right\rangle$ is the sequence of edges of a path, then $\left\langle\hat{Y}_{\pi 1}, \hat{Y}_{\pi 2}\right\rangle,\left\langle\hat{Y}_{\pi 2}, \hat{Y}_{\pi 3}\right\rangle, \ldots,\left\langle\hat{Y}_{\pi(k-1)}, \hat{Y}_{\pi k}\right\rangle$ are all sequences of edges of paths. By iteration of the case $k=2$, we see that the sequence of edges of a path of arbitrary length $\geq 2$ uniquely determines the initial and terminal vertices.

To prove the second assertion, let $\left\langle\hat{Y}_{\pi 1}, \ldots, \hat{Y}_{\pi k}\right\rangle$ be the sequence of edges of a path of $\Gamma(\delta)$. It is immediate from the first assertion that $Y_{\pi 1} \ldots Y_{\pi k}=$ 
$\pm e_{i_{1} j_{k}} \neq 0$, where $i_{1}$ and $j_{k}$ are the initial and terminal vertices of the path determined by $\left\langle\hat{Y}_{\pi 1}, \cdots, \hat{Y}_{\pi k}\right\rangle$. This correspondence $\left\langle\hat{Y}_{\pi 1}, \cdots, \hat{Y}_{\pi k}\right\rangle \leftrightarrow Y_{\pi 1} \cdots Y_{\pi k}$ is easily seen to be the desired $1: 1$ correspondence between paths of $\Gamma(\mathcal{S})$ of length $k$ and nonzero monomials in $Y_{1}, \cdots, Y_{v}$ of degree $k$. Q.E.D.

For any nonzero monomial in $Y_{1}, \ldots, Y_{v}$, the path corresponding to this monomial will be called the associated path. Let the $\Gamma(\delta)$-degree of a vertex of $\Gamma(\delta)$ be the number of edges (in $\Gamma(\delta)$ ) incident to it, with double loop count (i.e. $\{1,1\}$ is counted twice in the $\Gamma(\delta)$-degree of the vertex 1$)$. When the graph under consideration is clear, we shall denote $\Gamma(\mathcal{S})$-degree merely as the degree. If $S_{v}\left(Y_{1}, \cdots, Y_{v}\right) \neq 0$ then there is a nonzero monomial $Y_{\pi 1} \cdots Y_{\pi v}$, implying $\Gamma(\mathcal{S})$ has a path of length $v$. Since all intermediate vertices in this path are in pairs, only the end vertices can have odd degree. It follows that exactly zero or two vertices have odd degree. If all vertices have even degree then the end vertices of any path of length $v$ must be the same. Such a path is called an Euler path and its associated monomial has value $\pm e_{i i}$ where $i$ is the end vertex.

Lemma 9. Let $\mathcal{S}=\left\{Y_{1}, \cdots, Y_{v}\right\} \subseteq B_{u}^{-} \cup\{1,1\}, v \geq 2$. If $S_{v}\left(Y_{1}, \cdots, Y_{v}\right) \neq 0$ then one of the following two situations bolds:

(a) Each vertex $i$ of $\Gamma(\mathcal{S})$ bas even degree $d_{i}$. If $v$ is odd then $S_{v}\left(Y_{1}, \cdots, Y_{v}\right)=$ $\beta \Sigma_{i=1}^{u} d_{i} e_{i i}$, suitable $\beta$ in $\mathbf{Z}$.

(b) Two vertices $i$ and $j$ have odd degree, and for some $a$ in $Z$, $S_{v}\left(Y_{1}, \cdots, Y_{v}\right)=a\left(e_{i j} \pm e_{j i}\right)$.

Proof. (a) Suppose all vertices have even degree. Then all paths of length $v$ are Euler. Call two paths equivalent if they differ by a cyclic permutation. Consider one of these equivalence classes of Euler paths. A representative path with end vertices $r$ has value $\gamma e_{r r}$ where $\gamma= \pm 1$, and it is easy to see that $\gamma$ is an invariant of the class. Let us say the class has positive (negative) type if $\gamma=$ $+1(\gamma=-1)$.

Let $i$ be an arbitrary vertex. The intermediate vertices of any path are in pairs, so it is easy to see there are $\left(d_{i} / 2\right)$ Euler paths with end vertices $i$ in any equivalence class. These $\left(d_{i} / 2\right)$ paths have the same value, which is $\gamma e_{i i}, \gamma$ as above. Since any cyclic permutation on $(1, \cdots, v)$ has sign +1 (because $v$ is odd), we conclude that $e_{i i} s_{v}\left(Y_{1}, \ldots, Y_{v}\right) e_{i i}=\delta\left(d_{i} / 2\right) e_{i i}$, where $\delta$ is the number of classes of positive type minus the number of classes of negative type. Thus $S_{v}\left(Y_{1}, \cdots, Y_{v}\right)=(\delta / 2) \Sigma_{i=1}^{u} d_{i} e_{i i}$. Now the number of classes of Euler paths is even, because we can pair the Euler path whose sequence of edges is $\left\langle\hat{Y}_{\pi 1}, \cdots, \hat{Y}_{\pi \nu}\right\rangle$ with its opposite path whose sequence of edges is $\left\langle\hat{Y}_{\pi \nu}, \cdots, \hat{Y}_{\pi 1}\right\rangle$; this pairing induces a pairing of equivalence classes, showing in turn that $\delta$ is 
even. Let $\beta=\delta / 2$. Then $\beta \in Z$ and $S_{v}\left(Y_{1}, \cdots, Y_{\nu}\right)=\beta \sum_{i=1}^{u} d_{i} e_{i i}$.

(b) Suppose some vertex $i$ has odd degree. Then some other vertex $j$ has odd degree and $i, j$ are the end vertices of any path of length $v$. Thus, the associated monomials have value $\pm e_{i j}$ or $\pm e_{j i}$, and $S_{v}\left(Y_{1}, \ldots, Y_{v}\right)=\alpha e_{i j}+\beta e_{j i}$, suitable $\alpha, \beta$ in Z. But $S_{v}\left(Y_{1}, \cdots, Y_{v}\right)$ is either symmetric or antisymmetric by Lemma 4 , so $\beta= \pm \alpha$. Q.E.D.

Now let $\mathcal{S}=\left\{A_{1}, \cdots, A_{2 n-1}\right\}$, where $A_{1}, \cdots, A_{2 n-2} \in B_{n}^{-}$and $A_{2 n-1}=e_{11}$. The sum of all degrees in $\Gamma(\mathcal{S})$ is $2(2 n-1)=4 n-2$. Since $\Gamma(\mathcal{S})$ has $n$ vertices, some vertex has degree $\leq 3$. We consider the following two cases:

Case I. Some vertex has degree 0,1 , or 3 .

Case II. Some vertex has degree 2 , and no vertex has degree 0,1 , or 3 .

3. Case 1. Assume the vertex $j$ has degree 0,1 , or 3. First suppose $j$ has degree 0 . Then $j$ does not occur in $A_{1}, \cdots, A_{2 n-1}$. Hence we may view $A_{1}, \cdots$, $A_{2 n-1}$ in $M_{n-1}(Z) . S_{2 n-1}$ is an identity of $M_{n-1}(Z)$ by the Amitsur-Levitzki theorem and (1), so $S_{2 n-1}\left(A_{1}, \cdots, A_{2 n-1}\right)=0$ and we are done.

Suppose $j$ has degree 1 . We may assume $j$ is incident to $\hat{A}_{1}$, in which case $e_{j j} A_{r}=0$ for $r>1$. Thus, by (1),

$$
e_{j j} S_{2 n-1}\left(A_{1}, \ldots, A_{2 n-1}\right)=e_{j j} A_{1} S_{2 n-2}\left(A_{2}, \ldots, A_{2 n-1}\right)=0
$$

by Amitsur-Levitzki, viewing $A_{2}, \cdots, A_{2 n-1}$ in $M_{n-1}(\mathrm{Z})$. This implies $S_{2 n-1}\left(A_{1}, \cdots, A_{2 n-1}\right)=0$ by Lemma $9(\mathrm{~b})$.

Thus, we may assume $j$ has degree 3 . If $j=1$ then $j$ occurs twice in $A_{2 n-1}$, so $j$ occurs in only one other $A_{r}$, which we may assume is $A_{1}$. Then it is clear

$$
e_{j j} S_{2 n-1}\left(A_{1}, \cdots, A_{2 n-1}\right)=e_{j j} A_{2 n-1} A_{1} S_{2 n-2}\left(A_{2}, \cdots, A_{2 n-2}\right)=0
$$

by $\mathfrak{L}_{0}(n-1,2(n-1), 0)$, viewing $A_{2}, \cdots, A_{2 n-2}$ in $M_{n-1}^{-}(\mathrm{Z})$ (since $j$ does not occur in the se matrices). Hence $S_{2 n-1}\left(A_{1}, \cdots, A_{2 n-1}\right)=0$ by Lemma 9 (b).

So we may assume $j \neq 1$. In particular we may assume that $j$ occurs in $A_{1}$, $A_{2}, A_{3}$. By the Amitsur-Levitzki theorem we get $S_{2 n}\left(e_{j j}, A_{1}, \cdots, A_{2 n-1}\right)=0$, so expanding by (1) yields

$$
\begin{aligned}
0= & e_{j j} S_{2 n}\left(e_{j j}, A_{1}, \cdots, A_{2 n-1}\right) \\
= & e_{j j} e_{j j} S_{2 n-1}\left(A_{1}, \ldots, A_{2 n-1}\right)-e_{j j} A_{1} S_{2 n-1}\left(e_{j j}, A_{2}, \ldots, A_{2 n-1}\right) \\
& +e_{j j} A_{2} S_{2 n-1}\left(e_{j j}, A_{1}, A_{3}, \cdots, A_{2 n-1}\right) \\
& -e_{j j} A_{3} S_{2 n-1}\left(e_{j j}, A_{1}, A_{2}, A_{4}, \ldots, A_{2 n-1}\right)
\end{aligned}
$$

since $e_{j j} A_{r}=0$ unless $r=1,2$, or 3 . 
We shall now show $e_{j j} A_{1} S_{2 n-1}\left(e_{j j}, A_{2}, \cdots, A_{2 n-1}\right)=0$. Let $\hat{A}_{1}=\left\{i_{1}, j\right\}$, $\hat{A}_{2}=\left\{i_{2}, j\right\}, \hat{A}_{3}=\left\{i_{3}, j\right\}$. Any nonzero monomial of $e_{j j} A_{1} S_{2 n-1}\left(e_{j j}, A_{2}, \cdots, A_{2 n-1}\right)$ must be either of the form

$$
\pm e_{j j} A_{1} A_{\pi 4} \cdots A_{\pi r} A_{3} e_{j j} A_{2} A_{\pi(r+1)} \cdots A_{\pi(2 n-1)}
$$

or of the form

$$
\pm e_{j j} A_{1} A_{\pi 4} \cdots A_{\pi r} A_{2} e_{j j} A_{3} A_{\pi(r+1)} \cdots A_{\pi(2 n-1)}
$$

where $\pi$ is a permutation of $(4, \cdots, 2 n-1)$. Thus

$$
\begin{aligned}
e_{j j} A_{1} S_{2 n-1}\left(e_{j j}, A_{2}, \cdots, A_{2 n-1}\right) & \\
= & e_{j j} A_{1}\left(S_{2 n-1}\left(e_{j j}, A_{2}, \ldots, A_{2 n-1} ; A_{3} e_{j j} A_{2}\right)\right. \\
& \left.\quad+S_{2 n-1}\left(e_{i j}, A_{2}, \ldots, A_{2 n-1} ; A_{2} e_{i j} A_{3}\right)\right) \\
= & e_{j j} A_{1}\left(S_{2 n-1}\left(A_{3}, e_{j j}, A_{2}, A_{4}, \ldots, A_{2 n-1} ; A_{3} e_{i j} A_{2}\right)\right. \\
& \left.+S_{2 n-1}\left(A_{3}, e_{i j}, A_{2}, A_{4}, \ldots, A_{2 n-1} ; A_{2} e_{j j} A_{3}\right)\right) \\
= & e_{j j} A_{1} S_{2 n-3}\left(\tilde{A}, A_{4}, \ldots, A_{2 n-1}\right)
\end{aligned}
$$

by Lemma 7, where $\tilde{A}=\sim_{\sim_{3}} e_{j j} A_{2}-A_{2} e_{j i} A_{3} \in B_{n}^{-} \cdot$ In fact, $\tilde{A}= \pm\left(e_{i_{2} i_{3}}-e_{i_{3}{ }_{2}}\right)$, so $j$ does not occur in $\tilde{A}, A_{4}, \cdots, A_{2 n-1}$, which we may therefore view in $M_{n-1}^{-}(\mathrm{Z})$. Hence $S_{2 n-3}\left(\tilde{A}, A_{4}, \cdots, A_{2 n-1}\right)=0$ by $\mathfrak{L}_{0}(n-1,2(n-1)-1,1)$, so $e_{j j} A_{1} s_{2 n-1}\left(e_{j j}, A_{2}, \cdots, A_{2 n-1}\right)=0$. Analogous arguments show

$$
\begin{aligned}
e_{j i} A_{2} S_{2 n-1}\left(e_{j j}, A_{1}, A_{3}, \cdots, A_{2 n-1}\right) & =0 \text { and } \\
e_{i j} A_{3} S_{2 n-1}\left(e_{j j}, A_{1}, A_{2}, A_{4}, \cdots, A_{2 n-1}\right) & =0
\end{aligned}
$$

so (2) implies $0=e_{j j} e_{j j} S_{2 n-1}\left(A_{1}, \cdots, A_{2 n-1}\right)=e_{j j} S_{2 n-1}\left(A_{1}, \cdots, A_{2 n-1}\right)$. We conclude by Lemma 9 (b) that $S_{2 n-1}\left(A_{1}, \cdots, A_{2 n-1}\right)=0$, so Case I has been taken care of.

\section{Reduction to $n$ even and introduction of the graph $\Gamma^{\prime}$.}

Lemma 10. For $u \geq 2, v \geq 1$, let $Y_{1}, \ldots, Y_{2 v} \in B_{u}^{-}$and $Y_{2 v+1}=e_{11}$. Suppose some vertex $i$ of $\Gamma\left(\left\{Y_{1}, \ldots, Y_{2 n+1}\right\}\right)$ has degree 2 . If $i=1$ then $S_{2 v+1}\left(Y_{1}, \cdots, Y_{2 v+1}\right)=0$. If $i \neq 1$ then there is a permutation $\pi$ of $(1, \cdots, 2 v)$ for which $i$ occurs in $Y_{\pi 1}$ and $Y_{\pi 2}$, and 


$$
\begin{aligned}
e_{i} S_{2 v+1}\left(Y_{1}, \ldots, Y_{2 v+1}\right) e_{i i} & \\
=(s g \pi) e_{i i}\left(Y_{\pi 2} S_{2 v-1}\right. & \left(Y_{\pi 3}, \ldots, Y_{\pi(2 v)}, Y_{2 v+1}\right) Y_{\pi 1} \\
& \left.-Y_{\pi 1} S_{2 v-1}\left(Y_{\pi 3}, \ldots, Y_{\pi(2 v)}, Y_{2 v+1}\right) Y_{\pi 2}\right) e_{i i}
\end{aligned}
$$

Proof. If $i=1$ then $i$ does not occur in $Y_{r}, 1 \leq r \leq 2 v$, so $Y_{r} Y_{2 v+1}=$ $Y_{2 v+1} Y_{r}=0$, implying $S_{2 v+1}\left(Y_{1}, \cdots, Y_{2 v+1}\right)=0$. If $i \neq 1$ then there is a permutation $\pi$ of $(1, \ldots, 2 v)$ for which $i$ occurs in $Y_{\pi 1}$ and $Y_{\pi 2}$. Clearly $e_{i i} Y_{r}=$ $Y_{r} e_{i i}=0$ for $r \geq 3$, so the rest of the lemma is an easy consequence of (1). Q.E.D.

Now suppose $n$ is odd. We claim tr $S_{2 n-1}\left(A_{1}, \cdots, A_{2 n-1}\right)=0$. This is trivial unless all vertices of $\Gamma(\mathcal{S})$ have even degree. But then some vertex $i$ has degree 2 , and by Lemma 10 we have a permutation $\pi$ of $(1, \ldots, 2 n-2)$ such that $i$ occurs in $A_{\pi 1}$ and $A_{\pi 2}$. Viewing $A_{\pi 3}, \cdots, A_{\pi(2 n-1)}, A_{2 n-1}$ in $M_{n-1}(\mathrm{Z})$, we have by $\mathscr{L}_{0}(n-1,2(n-1)-1,1)$ that $S_{2(n-1)-1}\left(A_{\pi 3}, \cdots, A_{\pi(2 n-2)}, A_{2 n-1}\right)=0$. Hence $e_{i i} S_{2 n-1}\left(A_{1}, \cdots, A_{2 n-1}\right) e_{i i}=0$ by Lemma 10 , so $S_{2 n-1}\left(A_{1}, \cdots, A_{2 n-1}\right)=$ 0 by Lemma $9(\mathrm{a})$, establishing the claim. Hence, we have $\mathfrak{L}_{0}(n, 2 n-1,1)$ and $\mathfrak{L}_{0}(n, 2 n-1,0)$ by Lemma 6 , and are done for $n$ odd.

Thus we shall assume for the remainder of the proof of Theorem 1 that $n$ is even, and we shall prove $\mathfrak{L}_{0}(n+1,2(n+1)-1,0)$. Since $(n+1)$ is odd, it is enough to show for all $A_{1}, \cdots, A_{2 n}$ in $B_{n+1}^{-}$and $A_{2 n+1}=e_{11}$, that $\operatorname{tr} S_{2(n+1)-1}\left(A_{1}, \cdots, A_{2 n+1}\right)=0$. Let $\mathcal{S}^{\prime}=\left\{A_{1}, \cdots, A_{2 n+1}\right\}$ and let $\Gamma$-degree of an index denote its degree in $\Gamma\left(\mathcal{S}^{\prime}\right)$. Clearly tr $S_{2 n+1}\left(A_{1}, \cdots, A_{2 n+1}\right)=0$ if any index has odd $\Gamma$-degree (cf. Lemma 9), so we may assume that all indices have even $\Gamma$-degree.

Consider the edges of $\Gamma\left(\mathcal{S}^{\prime}\right)$ having an incident vertex of $\Gamma$-degree 2 . The set of all such edges, together with all their incident vertices, forms a subgraph $\Gamma^{\prime}$ of $\Gamma\left(\mathcal{S}^{\prime}\right)$. Any index which is not a vertex of $\Gamma^{\prime}$ will be said to have $\Gamma^{\prime}$-degree 0 . By definition of $\Gamma^{\prime}$, any edge of $\Gamma^{\prime}$ is incident to a vertex of $\Gamma$-degree 2 . On the other hand, by Lemma $9(a), s_{2 n+1}\left(A_{1}, \cdots, A_{2 n+1}\right)=\alpha \Sigma_{i=1}^{n+1} d_{i} e_{i i}$, some $\alpha$ in $\mathbf{Z}$. We are done if $a=0$, and otherwise there is the following result.

Lemma 11. If $a \neq 0$ then each edge of $\Gamma^{\prime}$ is incident to a vertex of $\Gamma$-degree $\geq 6$; in otber words, no vertex of $\Gamma^{\prime}$ bas $\Gamma$-degree 4, and no two vertices of $\Gamma$. degree 2 are incident to the same edge.

Proof. Suppose we have an edge $\hat{A}_{r}=\{i, k\}, i$ of $\Gamma$-degree 2 and $k$ of $\Gamma$-degree $<6$. We shall see how Lemma 10 implies $\alpha=0$. This is immediate if $i=k$, so assume $i \neq k$ and let $\hat{A}_{s}$ be the other edge of $\Gamma\left(\mathcal{S}^{\prime}\right)$ incident to $i$. For conve- 
nience, assume $r=1$ and $s=2$. Then in $\Gamma\left(\left\{A_{3}, \cdots, A_{2 n+1}\right\}\right) i$ has degree 0 and $k$ has degree 1 or 3. Hence we may view $A_{3}, \cdots, A_{2 n+1}$ in $M_{n}(\mathrm{Z})$, and $S_{2 n-1}\left(A_{3}, \cdots, A_{2 n+1}\right)=0$ by Case I. Therefore $\alpha=0$ by Lemma 10. Q.E.D.

Definition. The weight of a vertex of $\Gamma\left(\mathcal{S}^{\prime}\right)$ is its $\Gamma$-degree minus its $\Gamma^{\prime}$-degree.

Lemma 13. For each $d>0$ let $u_{d}$ be the number of vertices of $\Gamma$-degree $d$ and let $w_{d}$ be the sum of the weights of all vertices of $\Gamma$-degree d. If $a \neq 0$ then $\Sigma_{d \geq 6} w_{d}=4 \Sigma_{d \geq 6} u_{d}-2$.

Proof. Clearly $u_{d}=0$ for $d$ odd. $\Sigma_{d} u_{d}$ is the number of vertices of $\Gamma(\delta)$, which is $n+1$, so $0=\Sigma_{d} u_{d}-(n+1)$. Likewise, $\Sigma_{d} d u_{d}$ is the sum of all the $\Gamma$-degrees, which is $4 n+2$ since there are $2 n+1$ edges, each adjacent to two vertices. Thus

$$
\begin{aligned}
0 & =\sum_{d} d u_{d}-(4 n+2) \\
& =\left(\sum_{d} d u_{d}-(4 n+2)\right)-4\left(\sum_{d} u_{d}-(n+1)\right)=\sum_{d}(d-4) u_{d}+2 .
\end{aligned}
$$

This means $2 u_{2}=2+\Sigma_{d \geq 6}(d-4) u_{d}$. Now Lemma 12 says that each edge of $\Gamma^{\prime}$ is incident to one vertex of $\Gamma$-degree 2 and one vertex of $\Gamma$-degree $\geq 6$. Thus, the sum of $\Gamma^{\prime}$-degree of all vertices of $\Gamma$-degree $\geq 6=$ the sum of $\Gamma^{\prime}$-degrees of all vertices of $\Gamma$-degree $2=2 u_{2}$, since each vertex of $\Gamma$-degree 2 has $\Gamma^{\prime}$-degree 2 by definition of $\Gamma^{\prime}$. By definition of weight,

$$
\begin{aligned}
\sum_{d \geq 6} w_{d}= & (\text { sum of } \Gamma \text {-degrees of all vertices of } \Gamma \text {-degree } \geq 6) \\
& -\left(\text { sum of } \Gamma^{\prime} \text {-degrees of all vertices of } \Gamma \text {-degree } \geq 6\right) \\
= & \sum_{d \geq 6} d u_{d}-2 u_{2}=\sum_{d \geq 6} d u_{d}-\left(2+\sum_{d \geq 6}(d-4) u_{d}\right) \\
= & 4 \sum_{d \geq 6} u_{d}-2 .
\end{aligned}
$$

Suppose every vertex of $\Gamma$-degree $2 d$ has weight $\geq d$, all $d \geq 3$. In particular every vertex of $\Gamma$-degree $\geq 8$ has weight $\geq 4$, and $4 \Sigma_{d \geq 6} u_{d}-2=\Sigma_{d \geq 6} w_{d}=$ $w_{6}+\Sigma_{d \geq 8} w_{d} \geq w_{6}+4 \Sigma_{d \geq 8} u_{d}$. In this case $4 u_{6}-2 \geq w_{6}$; since each vertex of $\Gamma$-degree 6 is assumed to have weight $\geq 3$, we conclude that two vertices of $\Gamma$. degree 6 have weight 3.

Thus we are reduced to the following two subcases of Case II:

Subcase A. Some vertex of $\Gamma$-degree $2 d$ has weight $<d$, for some $d \geq 3$.

Subcase B. Two vertices of $\Gamma$-degree 6 have weight 3 . 


\section{Reduction to Subcase B.}

Lemma 12. Let $Y_{1}, \cdots, Y_{2 k} \in B_{k+1}^{-}, k \leq n$, and let $Y_{2 k+1}=e_{11} \in B_{k+1}^{+}$. For $m \geq 1$, suppose there exist indices $i_{1}, \cdots, i_{2 m}$, each of degree 2 in $\Gamma\left(\left\{Y_{1}, \cdots, Y_{2 k+1}\right\}\right)$, such that $i_{1}$ occurs in $Y_{1}$ and $Y_{2}, i_{2}$ occurs in $Y_{3}$ and $Y_{4}$, $\cdots, i_{2 m}$ occurs in $Y_{4 m-1}$ and $Y_{4 m}$. For $1 j \leq m$ we define the monomials $W_{j}=Y_{4 j-3} Y_{4 j-2} Y_{4 j-1} Y_{4 j}$ and $W_{j}^{\prime}=Y_{4 j} Y_{4 j-1} Y_{4 j-2} Y_{4 j-3}$. Then

$$
\sum s_{2 k+1}\left(Y_{1}, Y_{2}, \ldots, Y_{2 k+1} ; v_{1}, \ldots, v_{m}\right)=0,
$$

the sum taken over the $2^{m}$ terms obtained by specializing $V_{j}$ to $W_{j}$ or $W_{j}^{\prime}, 1 \leq$ $j \leq m$.

Proof. The lemma will be proved by induction on $m$. Let $\Sigma$ be an abbreviation for $\Sigma S_{2 k+1}\left(Y_{1}, \cdots, Y_{2 k+1} ; V_{1}, \cdots, V_{m}\right)$, the sum taken over the $2^{m}$ terms obtained by specializing $V_{j}$ to $W_{j}$ or $W_{j}^{\prime}, 1 \leq j \leq m . \Sigma=\Sigma_{1}+\Sigma_{2}+\Sigma_{3}, \Sigma_{1}$ being the sum of those monomials of $\Sigma$ ending with $W_{1}^{\prime}, \Sigma_{2}$ being the sum of those monomials of $\Sigma$ starting with $W_{1}$, and $\Sigma_{3}$ being the sum of those monomials of $\Sigma$ neither starting with $W_{1}$ nor ending with $W_{1}^{\prime}$. We shall show (i) $\Sigma_{1}=0$; (ii) $\Sigma_{2}=$ 0; (iii) $\Sigma_{3}=0$.

(i) $\Sigma_{1}=\Sigma S_{2 k-3}\left(Y_{5}, Y_{6}, \cdots, Y_{2 k+1} ; V_{2}, \cdots, V_{m}\right) W_{1}^{\prime}$, the sum taken over the the $2^{m-1}$ terms obtained by specializing $V_{j}$ to $W_{j}$ or to $W_{j}^{\prime}, 2 \leq j \leq m$. Now $i_{1}$ and $i_{2}$ occur only in $Y_{1}, Y_{2}, Y_{3}, Y_{4}$, so we may view $Y_{5}, \cdots, Y_{2 k}$ in $B_{k-1}^{-}$. If $m=1$ then $S_{2(k-1)-1}\left(Y_{5}, \cdots, Y_{2 k+1}\right)=0$ by $£_{0}(k-1,2(k-1)-1,1)$, so $\Sigma_{1}=0$. On the other hand, if $m>1$, then $\Sigma S_{2(k-2)+1}\left(Y_{5}, \cdots, Y_{2 k+1} ; V_{2}, \cdots, V_{m}\right)=0$ by induction on $m$, so $\Sigma_{1}=0$. Thus $\Sigma_{i}=0$ for all $m \geq 1$.

(ii) $\Sigma_{2}=0$ is proved in a manner analogous to (i).

(iii) Consider a typical monomial of $\Sigma_{3}$. This has either a submonomial $Y_{r} W_{1}$ or a submonomial $W_{1}^{\prime} Y_{r}, 5 \leq r \leq 2 k+1$. To prove $\Sigma_{3}=0$ it suffices to prove for each $r, 5 \leq r \leq 2 k+1$, that the sum of those monomials of $\Sigma$ containing either the submonomial $Y_{r} W_{1}$ or the submonomial $W_{1}^{\prime} Y_{r}$ is 0 .

If $4 m<r \leq 2 k+1$ then this sum is (by Lemma 7)

$$
\sum s_{2 k-3}\left(Y_{5}, \ldots, Y_{r-1}, \tilde{Y}_{r}, Y_{r+1}, \ldots, Y_{2 k+1} ; V_{2}, \ldots, v_{m}\right) \text {, }
$$

summed over the $2^{m-1}$ specializations of $V_{j}$ to $W_{j}$ or $W_{j}^{\prime}, 2 \leq j \leq m$, where $\tilde{Y}_{r}=Y_{r} W_{1}+W_{1}^{\prime} Y_{r}$ is in $B_{k+1}^{-}$if $r<2 k+1$ and is in $B_{k+1}^{+}$if $r=2 k+1$. But the indices $i_{1}$ and $i_{2}$ do not occur in $Y_{5}, \cdots, Y_{2 k+1}$, or in $\tilde{Y}_{r}$, so we see

$$
\sum s_{2 k-3}\left(Y_{5}, \ldots, Y_{r-1}, \tilde{Y}_{r}, Y_{r+1}, \ldots, Y_{2 k+1} ; V_{2}, \ldots, V_{m}\right)=0 \text {, }
$$


by $\mathscr{L}_{0}(k-1,2(k-1)-1,1)$ if $m=1$, by induction (on $m$ ) if $m>1$.

On the other hand, suppose $5 \leq r \leq 4 m$. Then $Y_{r}$ is in $W_{j}$ or $W_{j}^{r}$, some $j \geq 2$; it follows that there are no nonzero monomials of $\Sigma$ containing the submonomial ${ }^{-}{ }_{r} W_{1}$ or the submonomial $W_{1}^{\prime} Y$, unless either $r=4 j-3$ or $r=4 j$. Let us assume $r=4 j-3$ (the other situation is analogous). Then all nonzero monomials of $\Sigma$ containing $Y_{r} W_{1}$ or $W_{1}^{\prime} Y_{r}$ must in fact contain $W_{j}^{\prime} W_{1}$ or $W_{1}^{\prime} W_{j}$. Let $\tilde{Y}_{r}=Y_{r} W_{1}+$ $W_{1}^{\prime} Y_{r} \tilde{Y}_{r} \in B_{k+1}^{-}$and is incident to the index $i_{2 j-1}$ since $r=4 j-3$. But $i_{2 j-1}$ is of degree 2 in $\Gamma\left(\left\{Y_{1}, \cdots, Y_{2 k+1}\right\}\right)$, occurring in $Y_{4 j-3}$ and $Y_{4 j-2}$, so it is clear that $W_{j}^{\prime} W_{1}=Y_{4 j} Y_{4 j-1} Y_{4 j-2} \widetilde{Y}_{r}$ and $W_{1}^{\prime} W_{j}=\tilde{Y}_{r} Y_{4 j-2} Y_{4 j-1} Y_{4 j}$. Thus if we let

$$
\widetilde{W}_{j}=\tilde{Y}_{r} Y_{4 j-2} Y_{4 j-1} Y_{4 j} \text { and } \widetilde{W}_{j}^{\prime}=Y_{4 j} Y_{4 j-1} Y_{4 j-2} \widetilde{Y}_{r}
$$

then the sum of those monomials of $\Sigma$ containing $Y_{r} W_{1}$ or $W_{1}^{\prime} Y_{r}$ is

$$
\sum S_{2 k-3}\left(Y_{5}, \ldots, \widetilde{Y}_{r}, \ldots, Y_{2 k+1} ; v_{2}, \ldots, \widetilde{V}_{j}, \ldots, v_{m}\right) \text {, }
$$

summed over the $2^{m-1}$ specializations of $V_{1}$ to $W_{1}$ or $W_{1}^{\prime}, \cdots, \widetilde{V}_{j}$ to $\widetilde{W}_{j}$ or $\tilde{W}_{j}^{\prime}, \cdots, V_{m}$ to $W_{m}$ or $W_{m}^{\prime}$. But the indices $i_{1}$ and $i_{2}$ do not occur in $Y_{5}, \cdots$, $\tilde{Y}_{r}, \cdots, Y_{2 k+1}$, so we see

$$
\sum s_{2 k-3}\left(Y_{5}, \ldots, \widetilde{Y}_{r}, \ldots, Y_{2 k+1} ; v_{2}, \ldots, \widetilde{V}_{j}, \ldots, v_{m}\right)=0
$$

by induction. Q.E.D.

We can now dispose of subcase $A$. Namely, assume that the vertex $i$ has $\Gamma$ degree $2 d$, and that at least $(d+1)$ edges incident to $i$ are of the form $\left\{i_{\mu}, i\right\}, i_{\mu}$ an index of $\Gamma$-degree $2,1 \leq \mu \leq d+1$. Since $n \geq 2 d$ there certainly exists an index $k \notin\left\{i, i_{1}, \cdots, i_{d+1}\right\}$. By Lemma 9 , in order to show $a=0$ it is enough to show $e_{k k} S_{2 n+1}\left(A_{1}, \cdots, A_{2 n+1}\right) e_{k k}=0$, which we now claim. Indeed, for any nonzero monomial there is an associated Euler path in $\Gamma\left(\mathcal{S}^{\prime}\right)$ with end vertices $k$. The vertex $i$ can only be intermediate in this path, from which we conclude (even if $i=1$ ) that for some $r, s, 1 \leq r<s \leq d+1$, the edges $\left\{i, i_{r}\right\}$ and $\left\{i, i_{s}\right\}$ are adjacent. Now $\left\{i, i_{r}\right\}$ is adjacent to an edge $\left\{i_{r}, j_{1}\right\}$, and $\left\{i_{,} i_{s}\right\}$ is adjacent to an edge $\left\{i_{s}, j_{2}\right\}$, for suitable $j_{1}$ and $j_{2}$. Let $W_{r s}$ be the submonomial associated with the subpath $\left\{\left\langle\left\{j_{1}, i_{r}\right\},\left\{i_{r}, i\right\},\left\{i, i_{s}\right\},\left\{i_{s}, j_{2}\right\}\right\rangle, j_{1}, j_{2}\right\}$, and let $W_{r s}^{\prime}$ be the submonomial associated with the reverse subpath. Then by Lemma 12 ,

$$
e_{k k} S_{n+1}\left(A_{1}, \ldots, A_{2 n+1} ; W_{r s}\right) e_{k k}+e_{k k} S_{2 n+1}\left(A_{1}, \ldots, A_{2 n+1} ; W_{r s}^{\prime}\right) e_{k k}=0 .
$$

Summing over all possible $w_{r s}, r<s$, we get $\Sigma e_{k k} s_{2 n+1}\left(A_{1}, \cdots, A_{2 n+1} ; W_{r s}\right)=0$, the sum taken over all $w_{r s}, r \neq s$. In this summation we have counted every nonzero monomial of $e_{k k} S_{2 n+1}\left(A_{1}, \cdots, A_{2 n+1}\right) e_{k k}$, but we have counted twice the 
monomials of $\Sigma e_{k k} S_{2 n+1}\left(A_{1}, \cdots, A_{2 n+1} ; W_{r_{1} s_{1}}, W_{r_{2} s_{2}}\right) e_{k k}$, summed over distinct $r_{1}, s_{1}, r_{2}, s_{2}$. But if $r_{1}, s_{1}, r_{2}, s_{2}$ are distinct then the edges of the paths associated with $W_{r_{1} s_{1}}$ and $W_{r_{2} s_{2}}$ are all distinct, so we see by using Lemma 12 again that $\Sigma e_{k k} S_{2 n+1}\left(A_{1}, \cdots, A_{2 n+1} ; W_{r_{1} s_{1}}, W_{r_{2} s_{2}}\right) e_{k k}=0$. Continuing in this way (using the inclusion-exclusion principle) we have

$$
\begin{aligned}
& e_{k k} S_{2 n+1}\left(A_{1}, \cdots, A_{2 n+1}\right) e_{k k} \\
&= \sum e_{k k} s_{2 n+1}\left(A_{1}, \ldots, A_{2 n+1} ; W_{r s}\right) e_{k k} \\
&-\sum e_{k k} s_{2 n+1}\left(A_{1}, \ldots, A_{2 n+1} ; W_{r_{1} s_{1}}, W_{r_{2} s_{2}}\right) e_{k k} \\
&+\sum e_{k k} s_{2 n+1}\left(A_{1}, \ldots, A_{2 n+1} ; W_{r_{1} s_{1}}, W_{r_{2} s_{2}}, w_{r_{3} s_{3}}\right) e_{k k} \pm \cdots 0
\end{aligned}
$$

by repeated applications of Lemma 12. This concludes the proof $a=0$ in subcase A.

6. Completion of proof of Theorem 1: Subcase B. Having reduced the proof of $\mathscr{\varrho}_{0}(n+1,2(n+1)-1,1)$ to subcase $B$, we may assume that two distinct vertices of $\Gamma$-degree 6 have weight 3. In particular there exists a vertex $i \neq 1$ of $\Gamma$-degree 6 and weight 3 (i.e. $i$ is not incident to $\hat{A}_{2 n+1}=$ $\{1,1\})$. Assume the following: $\hat{A}_{r}=\{i, r\}, 2 \leq r \leq 7$, the vertices 2,3 , and 4 each have $\Gamma$-degree 2 , and $\hat{A}_{1}$ is the other edge of $\Gamma\left(\delta^{\prime}\right)$ incident to the vertex 2 . By Lemma 9, in order to show $a=0$ it is enough to show

$$
e_{22} S_{2 n+1}\left(A_{1}, \cdots, A_{2 n+1}\right) e_{22}=0,
$$

and by Lemma 10 it is enough to show $S_{2 n-1}\left(A_{3}, \cdots, A_{2 n+1}\right)=0$. Since the index 2 occurs only in $A_{1}$ and $A_{2}$, we may in fact view $A_{3}, \cdots, A_{2 n+1}$ in $B_{n}^{+} \cup B_{n}^{-}$. For the remainder of the proof of Theorem 1 , let degree denote degree in $\Gamma\left(\left\{A_{3}, \cdots, A_{2 n+1}\right\}\right)$. Clearly $i$ has degree 5 (being incident to $\hat{A}_{3}, \hat{A}_{4}, \hat{A}_{5}, \hat{A}_{6}$, $\hat{A}_{7}$ ) so by Lemma 9 there is another vertex $k$ of odd degree and it is enough to show $e_{i i} S_{2 n-1}\left(A_{3}, \cdots, A_{2 n+1}\right) e_{k k}=0$. Using the Amitsur-Levitzki theorem and expanding by (1), we get

$$
\begin{aligned}
0= & e_{i i} S_{2 n}\left(e_{i i}, A_{3}, \ldots, A_{2 n+1}\right) e_{k k} \\
= & e_{i i} e_{i i} S_{2 n-1}\left(A_{3}, \ldots, A_{2 n+1}\right) e_{k k} \\
& -e_{i i} A_{3} S_{2 n-1}\left(e_{i i}, A_{4}, \ldots, A_{2 n+1}\right) e_{k k} \\
& +e_{i i} A_{4} S_{2 n-1}\left(e_{i i}, A_{3}, A_{5}, \ldots, A_{2 n+1}\right) e_{k k} \\
& -e_{i i} A_{5} S_{2 n-1}\left(e_{i i}, A_{3}, A_{4}, A_{6}, \ldots, A_{2 n+1}\right) e_{k k} \\
& +e_{i i} A_{6} S_{2 n-1}\left(e_{i i}, A_{3}, A_{4}, A_{5}, A_{7}, \ldots, A_{2 n+1}\right) e_{k k} \\
& -e_{i i} A_{7} S_{2 n-1}\left(e_{i i}, A_{3}, \ldots, A_{6}, A_{8}, \ldots, A_{2 n+1}\right) e_{k k} \cdot
\end{aligned}
$$


Now $e_{i i} e_{i i} S_{2 n-1}\left(A_{3}, \cdots, A_{2 n+1}\right) e_{k k}=e_{i i} S_{2 n-1}\left(A_{3}, \cdots, A_{2 n+1}\right) e_{k k}$. We also claim $e_{i i} A_{3} S_{2 n-1}\left(e_{i i}, A_{4}, \cdots, A_{2 n+1}\right) e_{k k}=0$ and

$$
e_{i i} A_{4} S_{2 n-1}\left(e_{i i}, A_{3}, A_{5}, \ldots, A_{2 n+1}\right) e_{k k}=0 .
$$

Indeed, the vertex 3 , having degree two, is incident to $\hat{A}_{3}$ and to one other edge, which we may assume is $\hat{A}_{g}$. Likewise, assume the vertex 4 , also having degree two, is incident to $\hat{A}_{4}$ and $\hat{A}_{9}$. Since the index 3 does not occur in $e_{i i}, A_{4}, \cdots$, $A_{7}, A_{9}, \cdots, A_{2 n+1}$, we may view these matrices in $M_{n-1}(\mathrm{Z})$, so

$$
\begin{aligned}
& e_{i i} A_{3} S_{2 n-1}\left(e_{i i}, A_{4}, \cdots, A_{2 n+1}\right) e_{k k} \\
& \quad=e_{i i} A_{3} A_{8} S_{2(n-1)}\left(e_{i i}, A_{4}, \ldots, A_{7}, A_{9}, \cdots, A_{2 n+1}\right) e_{k k}=0
\end{aligned}
$$

by the Amitsur-Levitzki theorem. Similarly,

$$
e_{i i} A_{4} S_{2 n-1}\left(e_{i i}, A_{3}, A_{5}, \cdots, A_{2 n+1}\right) e_{k k}=0 .
$$

Therefore, equation ( 3 ) becomes

$$
\begin{aligned}
e_{i i} S_{2 n-1}\left(A_{3}, \cdots,\right. & \left.A_{2 n+1}\right) e_{k k} \\
= & e_{i i} A_{5} S_{2 n-1}\left(e_{i i}, A_{3}, A_{4}, A_{6}, \ldots, A_{2 n+1}\right) e_{k k} \\
& -e_{i i} A_{6} S_{2 n-1}\left(e_{i i}, A_{3}, A_{4}, A_{5}, A_{7}, \ldots, A_{2 n+1}\right) e_{k k} \\
& +e_{i i} A_{7} S_{2 n-1}\left(e_{i i}, A_{3}, A_{4}, A_{5}, A_{6}, A_{8}, \ldots, A_{2 n+1}\right) e_{k k} .
\end{aligned}
$$

Let us analyze $e_{i i} A_{5} S_{2 n-1}\left(e_{i i}, A_{3}, A_{4}, A_{6}, \cdots, A_{2 n+1}\right) e_{k k}$. Since $e_{i i} A_{5}=$ $\pm e_{i 5}$, it is equivalent to look at $e_{i 5} S_{2 n-1}\left(e_{i i}, A_{3}, A_{4}, A_{6}, \cdots, A_{2 n+1}\right) e_{k k}$ each of whose nonzero monomials contains at least one of the following submonomials:

$$
\begin{array}{lll}
\text { (i) } A_{3} e_{i i} & \text { (ii) } e_{i i} A_{3} & \text { (iii) } A_{4} e_{i i} \\
\text { (iv) } e_{i i} A_{4} & \text { (v) } A_{6} e_{i i} A_{7} & \text { (vi) } A_{7} e_{i i} A_{6}
\end{array}
$$

We shall see that the sum of the monomials of $e_{i 5} S_{2 n-1}\left(e_{i i}, A_{3}, A_{4}, A_{6}, \cdots, A_{2 n+1}\right) e_{k k}$ of types (i) and (ii) (resp. (iii) and (iv), (v) and (vi)) is 0 . Since the only duplication in counting occurs when we count twice monomials containing $A_{3} e_{i i} A_{4}$ or $A_{4} e_{i i} A_{3}$, it will follow that

$$
\begin{aligned}
e_{i i} A_{5} S_{2 n-1}\left(e_{i i}, A_{3}, A_{4}, A_{6}, \ldots, A_{2 n+1}\right) e_{k k} \\
=e_{i i} A_{5}\left(S_{2 n-1}\left(e_{i i}, A_{3}, A_{4}, A_{6}, \ldots, A_{2 n+1} ; A_{3} e_{i i} A_{4}\right)\right. \\
\left.\quad+S_{2 n-1}\left(e_{i i}, A_{3}, A_{4}, A_{6}, \ldots, A_{2 n+1} ; A_{4} e_{i i} A_{3}\right)\right) e_{k k} .
\end{aligned}
$$

First we claim that

$$
\begin{aligned}
& e_{i 5}\left(S_{2 n-1}\left(e_{i i}, A_{3}, A_{4}, A_{6}, \ldots, A_{2 n+1} ; A_{3} e_{i i}\right)\right. \\
& \left.\quad+S_{2 n-1}\left(e_{i i}, A_{3}, A_{4}, A_{6}, \ldots, A_{2 n+1} ; e_{i i} A_{3}\right)\right) e_{k k}=0
\end{aligned}
$$


Well, it is clear that, since the index 3 occurs only in $A_{3}$ and $A_{8}$,

$$
\begin{aligned}
& e_{i 5}\left(S _ { 2 n - 1 } \left(e_{i i},\right.\right.\left.A_{3}, A_{4}, A_{6}, \cdots, A_{2 n+1} ; A_{3} e_{i i}\right) \\
&\left.+S_{2 n-1}\left(e_{i i}, A_{3}, A_{4}, A_{6}, \ldots, A_{2 n+1} ; e_{i i} A_{3}\right)\right) e_{k k} \\
&= e_{i 5}\left(S_{2 n-1}\left(e_{i i}, A_{3}, A_{4}, A_{6}, \ldots, A_{2 n+1} ; A_{8} A_{3} e_{i i}\right)\right. \\
&\left.\quad \quad \quad S_{2 n-1}\left(e_{i i}, A_{3}, A_{4}, A_{6}, \cdots, A_{2 n+1} ; e_{i i} A_{3} A_{8}\right)\right) e_{k k} \\
&=-e_{i 5} S_{2 n-3}\left(\tilde{A}_{3}, A_{4}, A_{6}, A_{7}, A_{9}, \ldots, A_{2 n+1}\right) e_{k k}
\end{aligned}
$$

by Lemma 7, where $\tilde{A}_{3}=e_{i i} A_{3} A_{8}-A_{8} A_{3} e_{i i}$ is an antisymmetric matrix. Moreover the index 3 does not occur in $A_{3}, A_{4}, A_{6}, A_{7}, A_{9}, \cdots, A_{2 n+1}$, so we may view these matrices in $M_{n-1}(\mathrm{Z})$. $B y \mathcal{Q}_{0}(n-1,2(n-1)-1,1)$,

$$
S_{2 n-3}\left(\widetilde{A}_{3}, A_{4}, A_{6}, A_{7}, A_{9}, \cdots, A_{2 n+1}\right)=0,
$$

yielding the desired result.

The analogous argument shows that

$$
\begin{aligned}
& e_{i 5}\left(S_{2 n-1}\left(e_{i i}, A_{3}, A_{4}, A_{6}, \ldots, A_{2 n+1} ; A_{4} e_{i i}\right)\right. \\
&\left.+S_{2 n-1}\left(e_{i i}, A_{3}, A_{4}, A_{6}, \ldots, A_{2 n+1} ; e_{i i} A_{4}\right)\right) e_{k k}=0 .
\end{aligned}
$$

Now we claim

$$
\begin{aligned}
& e_{i 5}\left(S_{2 n-1}\left(e_{i i}, A_{3}, A_{4}, A_{6}, \ldots, A_{2 n+1} ; A_{6} e_{i i} A_{7}\right)\right. \\
& \left.\quad+S_{2 n-1}\left(e_{i i}, A_{3}, A_{4}, A_{6}, \ldots, A_{2 n+1} ; A_{7} e_{i i} A_{6}\right)\right) e_{k k}=0 .
\end{aligned}
$$

Well, by Lemma 8, this equals $-e_{i 5} S_{2 n-3}\left(A_{3}, A_{4}, \tilde{A}_{6}, A_{8}, \cdots, A_{2 n+1}\right) e_{k k}$, where $\tilde{A}_{6}=A_{6} e_{i i} A_{7}-A_{7} e_{i i} A_{6}$. Since $i$ does not occur in $\tilde{A}_{6}, A_{8}, \cdots, A_{2 n+1}$, we see $A_{3}$ and $A_{4}$ are adjacent in any nonzero monomial (of

$$
\left.e_{i 5} S_{2 n-3}\left(A_{3}, A_{4}, \tilde{A}_{6}, A_{8}, \cdots, A_{2 n+1}\right) e_{k k}\right) \text {, }
$$

which must therefore contain either $A_{8} A_{3} A_{4} A_{9}$ or $A_{9} A_{4} A_{3} A_{8}$. Thus,

$$
\begin{aligned}
e_{i 5} S_{2 n-3}\left(A_{3}, A_{4}, \breve{A}_{6}, A_{8}, \ldots, A_{2 n+1}\right) e_{k k} \\
=e_{i 5}\left(S_{2 n-3}\left(A_{3}, A_{4}, \tilde{A}_{6}, A_{8}, \ldots, A_{2 n+1} ; A_{8} A_{3} A_{4} A_{9}\right)\right. \\
\left.\quad+S_{2 n-3}\left(A_{3}, A_{4}, A_{6}, A_{8}, \ldots, A_{2 n+1} ; A_{9} A_{4} A_{3} A_{8}\right)\right) e_{k k} .
\end{aligned}
$$

In order to prepare for the use of Lemma 12, let us replace the index $i$ in $A_{3}$ and $A_{4}$ by the index 1 , calling the results $\bar{A}_{3}$ and $\bar{A}_{4}$. Then the index $i$ does not occur in $\bar{A}_{3}, \bar{A}_{4}, \tilde{A}_{6}, A_{8}, \cdots, A_{2 n+1}$, so we may view these matrices in $M_{n-1}(\mathrm{Z})$. Since $A_{9} \bar{A}_{4} \bar{A}_{3} A_{8}=A_{9} A_{4} A_{3} A_{8}$ and $A_{8} \bar{A}_{3} \bar{A}_{4} A_{9}=A_{8} A_{3} A_{4} A_{9}$, we have 


$$
\begin{aligned}
S_{2 n-3}( & \left.A_{3}, A_{4}, \tilde{A}_{6}, A_{8}, \cdots, A_{2 n+1} ; A_{8} A_{3} A_{4} A_{9}\right) \\
& +S_{2 n-3}\left(A_{3}, A_{4}, \tilde{A}_{6}, A_{8}, \cdots, A_{2 n+1} ; A_{9} A_{4} A_{3} A_{8}\right) . \\
= & S_{2 n-3}\left(\bar{A}_{3}, \bar{A}_{4}, \tilde{A}_{6}, A_{8}, \cdots, A_{2 n+1} ; A_{8} \bar{A}_{3} \bar{A}_{4} A_{9}\right) \\
& +S_{2 n-3}\left(\bar{A}_{3}, \bar{A}_{4}, \tilde{A}_{6}, A_{8}, \cdots, A_{2 n+1} ; A_{9} \bar{A}_{4} \bar{A}_{3} A_{8}\right)=0
\end{aligned}
$$

by Lemma 12. Thus, $e_{i 5} S_{2 n-3}\left(A_{3}, A_{4}, \tilde{A}_{6}, A_{8}, \cdots, A_{2 n+1}\right) e_{k k}=0$, establishing the claim.

These three results show, as has already been observed, that

$$
\begin{aligned}
e_{i i} A_{5} S_{2 n-1}\left(e_{i i}, A_{3}, A_{4}, A_{6}, \ldots, A_{2 n+1}\right) e_{k k} \\
=e_{i i} A_{5}\left(S_{2 n-1}\left(e_{i i}, A_{3}, A_{4}, A_{6}, \ldots, A_{2 n+1} ; A_{3} e_{i i} A_{4}\right)\right. \\
\left.\quad+S_{2 n-1}\left(e_{i i}, A_{3}, A_{4}, A_{6}, \ldots, A_{2 n+1} ; A_{4} e_{i i} A_{3}\right)\right) e_{k k} .
\end{aligned}
$$

Analogously,

$$
\begin{aligned}
e_{i i} A_{6} S_{2 n-1}\left(e_{i i}, A_{3}, A_{4}, A_{5}, A_{7}, \ldots, A_{2 n+1}\right) e_{k k} \\
=e_{i i} A_{6}\left(S_{2 n-1}\left(e_{i i}, A_{3}, A_{4}, A_{5}, A_{7}, \ldots, A_{2 n+1} ; A_{3} e_{i i} A_{4}\right)\right. \\
\left.\quad+S_{2 n-1}\left(e_{i i}, A_{3}, A_{4}, A_{5}, A_{7}, \ldots, A_{2 n+1} ; A_{4} e_{i i} A_{3}\right)\right) e_{k k}
\end{aligned}
$$

and

$$
\begin{aligned}
& e_{i i} A_{7} S_{2 n-1}\left(e_{i i}, A_{3}, \cdots, A_{6}, A_{8}, \cdots, A_{2 n+1}\right) e_{k k} \\
&=e_{i i} A_{7}\left(S_{2 n-1}\left(e_{i i}, A_{3}, \cdots, A_{6}, A_{8}, \ldots, A_{2 n+1} ; A_{3} e_{i i} A_{4}\right)\right. \\
&\left.+S_{2 n-1}\left(e_{i i}, A_{3}, \ldots, A_{6}, A_{8}, \cdots, A_{2 n+1} ; A_{4} e_{i i} A_{3}\right)\right) e_{k k} .
\end{aligned}
$$

Thus, in the terms in the right hand side of (4), we may consider only those nonzero monomials containing the submonomial $A_{3} e_{i i} A_{4}$ or $A_{4} e_{i i} A_{3}$. But these are precisely the nonzero monomials of $e_{i i} S_{2 n}\left(e_{i i}, A_{3}, \cdots, A_{2 n+1}\right) e_{k k}$ which contain the submonomial $A_{3} e_{i i} A_{4}$ or $A_{4} e_{i i} A_{3}$. Therefore, (4) becomes

$$
\begin{aligned}
e_{i i} S_{2 n-1} & \left(A_{3}, \ldots, A_{2 n+1}\right) e_{k k} \\
= & -e_{i i}\left(S_{2 n-1}\left(e_{i i}, A_{3}, \ldots, A_{2 n+1} ; A_{3} e_{i i} A_{4}\right)\right. \\
& \left.\quad+S_{2 n}\left(e_{i i}, A_{3}, \ldots, A_{2 n+1} ; A_{4} e_{i i} A_{3}\right)\right) e_{k k} \\
= & -e_{i i} S_{2 n-4}\left(\tilde{A}, A_{5}, A_{6}, A_{7}, A_{10}, \ldots, A_{2 n+1}\right) e_{k k}
\end{aligned}
$$

by Lemma 7, where $\widetilde{A}=A_{8} A_{3} e_{i i} A_{4} A_{9}+A_{9} A_{4} e_{i i} A_{3} A_{8}$. But the indices 3 and 4 do not occur in $\tilde{A}, A_{5}, A_{6}, A_{7}, A_{10}, \cdots, A_{2 n+1}$. Thus

$$
S_{2(n-2)}\left(\tilde{A}, A_{5}, A_{6}, A_{7}, A_{10}, \cdots, A_{2 n+1}\right)=0
$$


by the Amitsur-Levitzki theorem. Therefore $e_{i i} S_{2 n-1}\left(A_{3}, \cdots, A_{2 n+1}\right) e_{k k}=0$, which disposes of subcase $B$.

Thus we have $\mathcal{L}_{0}(n+1,2(n+1)-1,1)$ for $n$ even. But then Lemma 6 yields $\mathfrak{L}_{0}(n, 2 n-1,1)$ and $\mathscr{E}_{0}(n, 2 n-1,0)$, finishing the proof of Theorem 1 .

7. Additional results when $p \neq 0$.

Theorem 2. $£_{2}(n, k, t)$ for $k \geq n+t$.

Proof. By hypothesis $+1=-1$. Thus all elements of $B_{n}^{-}$are symmetric, $S_{k}\left(A_{1}, \cdots, A_{k}\right)=\Sigma_{\pi} A_{\pi 1} \cdots A_{\pi k}$, and we need show only $S_{k}\left(A_{1}, \cdots, A_{k}\right)=0$ for all $A_{1}, \cdots, A_{k-t}$ in $B_{n}^{-}$and $A_{k-t+1}, \cdots, A_{k}$ in $\left\{e_{i i}, 1 \leq i \leq n\right\}$. Suppose all indices have even degree in $\Gamma\left(\left\{A_{1}, \cdots, A_{k-t}\right\}\right)$. Then any nonzero monomial $A_{\pi 1} A_{\pi 2} \cdots A_{\pi k}$ has value $e_{j j}$, suitable $j$. But then $e_{j j}=\left(A_{\pi 1} A_{\pi 2} \cdots A_{\pi k}\right)^{*}=$ $A_{\pi k} \cdots A_{\pi 2} A_{\pi 1}$, so $A_{\pi 1} A_{\pi 2} \cdots A_{\pi k}+A_{\pi k} \cdots A_{\pi 2} A_{\pi 1}=2 e_{j j}=0$. Summing over all such pairs of nonzero monomials, we see $S_{k}\left(A_{1}, \cdots, A_{k}\right)=0$.

Thus we may assume in particular that not every index has degree 2 in $\Gamma\left(\left\{A_{1}, \cdots, A_{k-t}\right\}\right)$. Since the sum of the degrees of $\Gamma\left(\left\{A_{1}, \cdots, A_{k-t}\right\}\right)$ is $2(k-t) \geq 2 n$, we see that some index $i$ must occur in at least three elements of $\left\{A_{1}, \cdots, A_{k-t}\right\}$. But this means that any path of length $k$ in $\Gamma\left(\left\{A_{1}, \cdots, A_{k}\right\}\right)$ must contain a closed subpath beginning and ending at $i$. Let $\hat{A}_{\pi 1} \hat{A}_{\pi 2} \cdots \hat{A}_{\pi k}$ be a typical path of length $k$ and let $\hat{A}_{\pi r} \ldots \hat{A}_{\pi s}$ be a closed subpath of maximal length beginning and ending at $i$. Then the reverse subpath $\hat{A}_{\pi s} \hat{A}_{\pi(s-1)} \cdots \hat{A}_{\pi r}$ also is closed with end vertices $i$, and $A_{\pi 1} \cdots A_{\pi(r-1)} A_{\pi r} \cdots A_{\pi s} A_{\pi(s+1)} \cdots$ $A_{\pi k}+A_{\pi 1} \cdots A_{\pi(r-1)} A_{\pi s} A_{\pi(s-1)} \cdots A_{\pi r} A_{\pi(s+1)} \cdots A_{\pi k}=2 A_{\pi 1} \cdots A_{\pi k}=0$. Summing over all such pairs of monomials yields $S_{k}\left(A_{1}, \cdots, A_{k}\right)=0$. Q.E.D.

Unfortunately this theorem does not say anything about identities for antisymmetric matrices, since in fact $M_{n}^{+}(F)$ is precisely the set of antisymmetric matrices of $M_{n}(F)$ when $F$ has characteristic 2. Nevertheless, defining $[X, Y]=$ $X Y-Y X$, we have

Corollary. Let $\operatorname{char}(F)=2$. Then $S_{n}\left(\left[A_{1}, A_{1}^{*}\right], \ldots,\left[A_{n}, A_{n}^{*}\right]\right)=0$ for all $A_{1}, \cdots, A_{n}$ in $M_{n}(F)$. Moreover, if $k \geq n$ then

$$
S_{k}\left(\left[A_{1}, A_{2}\right],\left[A_{3}, A_{4}\right], \ldots,\left[A_{2 n-1}, A_{2 n}\right], A_{2 n+1}, \cdots, A_{n+k}\right)=0
$$

for all $A_{1}, \cdots, A_{2 n+1}$ in $M_{n}^{+}(F)$.

Proof. Obviously, $\left[A, A^{*}\right] \in M_{n}^{-}(F)$ for all $A$ in $M_{n}(F)$, and $\left[A_{1}, A_{2}\right] \in M_{n}^{-}(F)$ for all $A_{1}, A_{2}$ in $M_{n}^{+}(F)$, so the corollary follows immediately from Theorem 2. Q.E.D. 
Examples given in the next section show that Theorems 1 and 2 are sharp. However, there still remains the question concerning $\mathscr{L}_{p}(n, k, t)$ for $p$ an odd prime. We shall see that no positive results can be adduced for $t>0$, so let us see what happens when $t=0$.

Proposition 1. $\mathscr{\complement}_{3}(4,5,0)$.

Proof. Te need to show that for all $A_{1}, \cdots, A_{5}$ in $M_{4}^{-}(F), F$ having characteristic $3, s_{5}\left(A_{1}, \cdots, A_{5}\right)=0$. Indeed, it suffices to consider $A_{1}, \cdots, A_{5}$ in $B_{4}^{-}$, which has only six distinct elements. Thus, permuting the indices if necessary, we may assume $A_{1}=e_{12}-e_{21}, A_{2}=e_{13}-e_{31}, A_{3}=e_{23}-e_{32}, A_{4}=$ $e_{2 \dot{4}}-e_{42}, A_{5}=e_{34}-e_{43}$, in which case $S_{5}\left(A_{1}, \cdots, A_{5}\right)=6 e_{32}-6 e_{23}=0$ in characteristic 3. Q.E.D.

Proposition 2. The following sentences are equivalent for $n$ even and $p \mid(2 n-1)$ :

(i) For all $A_{1}, \cdots, A_{2 n-1}$ in $B_{n+1}^{-}$such that all vertices of

$\Gamma\left(\left\{A_{1}, \cdots, A_{2 n-1}\right\}\right)$ bave even degree, $S_{2 n-1}\left(A_{1}, \cdots, A_{2 n-1}\right)=0$ in characteristic $p$.

(ii) $\mathcal{L}_{p}(n, 2 n-3,0)$.

(iii) $\mathcal{L}_{p}(n+1,2(n+1)-3,0)$.

(iv) $\mathfrak{Q}_{p}(n+1,2(n+1)-4,0)$.

Proof. (i) $\Rightarrow$ (iv) Clearly $(i) \Rightarrow \operatorname{tr} S_{2 n-1}\left(A_{1}, \cdots, A_{2 n-1}\right)=0$ for all $A_{1}, \cdots$, $A_{2 n-1}$ in $B_{n+1}^{-}$, hence for all $A_{1}, \cdots, A_{2 n-1}$ in $M_{n+1}^{-}$. Thus by Lemma 6(a), $\mathscr{L}_{p}(n+1,2 n-2,0)$.

(iv) $\Rightarrow$ (iii) Immediate by Lemma 1 (b).

(iv) $\Rightarrow$ (ii) Immediate by Lemma 2 .

(iii) $\Rightarrow$ (i) Obvious.

(ii) $\Rightarrow$ (i) Since the sum of the degrees of $\Gamma\left(\left\{A_{1}, \cdots, A_{2 n-1}\right\}\right)$ is $4 n-2$, whereas there are $(n+1)$ vertices, some vertex must have degree 2 . Hence, $(i)$ follows immediately from Lemmas 10 and 9. Q.E.D.

Corollary 1. $\mathscr{L}_{3}(5,7,0)$ and $\mathfrak{L}_{3}(5,6,0)$.

Proof. Immediate from Propositions 1 and 2.

Corollary $2 . \mathscr{L}_{s}(6,9,0), \mathfrak{L}_{s}(7,11,0)$, and $\mathcal{L}_{s}(7,10,0)$.

Proof. By Proposition 2, it suffices to show for any $A_{1}, \cdots, A_{2 n-1}$ in $B_{n+1}^{-}$, $n=6$, such that all vertices of $\Gamma\left(\left\{A_{1}, \cdots, A_{2 n-1}\right\}\right)$ have even degree, $S_{2 n-1}\left(A_{1}, \cdots, A_{2 n-1}\right)=0$ in characteristic 5 . We claim this is true if any edge is 
incident to two vertices of degree 2. Indeed, suppose $i_{1}$ and $i_{2}$ each have degree $2, i_{1}$ occurring in $A_{1}$ and $A_{2}$ and $i_{2}$ occurring in $A_{1}$ and $A_{3}$. To show $s_{2 n-1}\left(A_{1}, \cdots, A_{2 n-1}\right)=0$, it suffices to show (by Lemmas 10 and 9) $S_{2 n-3}\left(A_{3}, \cdots, A_{2 n-1}\right)=0$. But $i_{2}$ has degree 1 in $\Gamma\left(\left\{A_{3}, \cdots, A_{2 n-1}\right\}\right)$, so by Lemma 9 it suffices to show $0=e_{i_{2} i_{2}} S_{2 n-3}\left(A_{3}, \cdots, A_{2 n-1}\right)=$ $e_{i_{2} i_{2}} A_{3} S_{2 n-4}\left(A_{4}, \cdots, A_{2 n-1}\right)$. But $i_{1}$ and $i_{2}$ do not occur in $A_{4}, \cdots, A_{2 n-1}$, so we may view these matrices in $M_{n-1}^{-}\left(Z_{5}\right)$. Then $S_{2 n-4}\left(A_{4}, \cdots, A_{2 n-1}\right)=0$ by $\mathscr{L}_{5}(n-1,2(n-1)-2,0)$, establishing the claim.

So we may limit our attention to graphs without any edges incident to two vertices of degree 2, a complete nonisomorphic set (with 7 vertices and 11 edges) being

(a)

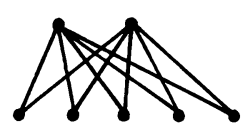

(c)

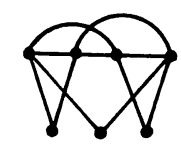

(b)

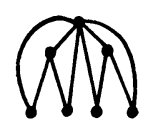

(d)

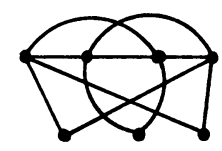

Each of these graphs corresponds to a set $\left\{A_{1}, \cdots, A_{11}\right\}$ of antisymmetric matrices such that $S_{11}\left(A_{1}, \cdots, A_{11}\right)=0$ in characteristic 5 . Interestingly, in each case $S_{11}\left(A_{1}, \cdots, A_{11}\right) \neq 0$ in characteristic 0 , so $(\mathrm{a})-(\mathrm{d})$ can be used to show that $\mathfrak{L}_{0}(n, 2 n-2,0)$ is sharp for $n=7$. Examples (a) and (c) have been generalized and explored in depth to show $\mathfrak{L}_{0}(n, 2 n-2,0)$ is sharp for all $n$ (example (a) was investigated independently by Rowen and Hutchinson, and example (c) was investigated by Owens [5]).

8. Counterexamples. We summarize the previous results (Lemma 1, Theorems 1 and 2, Proposition 1, and Corollaries 1 and 2 to Proposition 2):

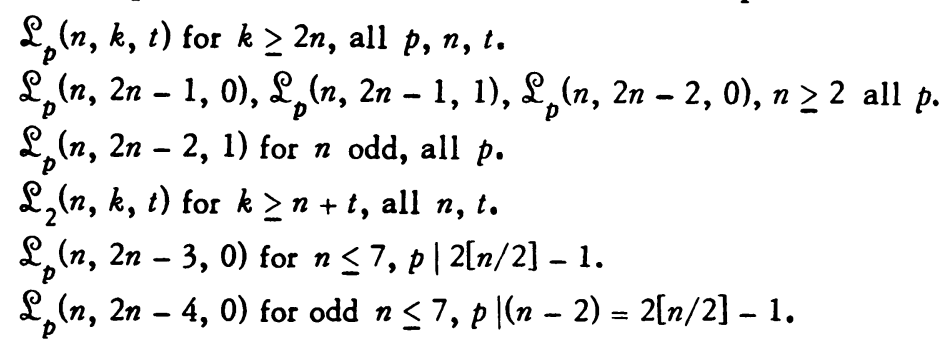

The purpose of this section is to show by example that these results are sharp for $p=0$ and $p=2$, and that for odd $p$ these results are sharp with the 
possible exception of $\mathscr{L}_{p}(n, 2 n-3,0)$ for $n \geq 8, p \mid 2[n / 2]-1$. Since the sentence which has evoked most interest is $\mathscr{L}_{0}(n, 2 n-3,0)$, we start with the most direct counterexample (known independently by Hutchinson).

Example 1. Let $A_{1}=e_{21}-e_{12}$. For $r \geq 1$ let $A_{2 r}=e_{1, r+2}-e_{r+2,1}$ and let $A_{2 r+1}=e_{r+2,2}-e_{2, r+2}$. For any $n \geq 2, A_{1}, \cdots, A_{2 n-3} \in B_{n}^{-}$;

$$
S_{2 n-3}\left(A_{1}, \cdots, A_{2 n-3}\right)=(-1)^{n / 2}(n-1) !\left(e_{12}-e_{21}\right)
$$

for $n$ even, and

$$
\begin{aligned}
& S_{2 n-3}\left(A_{1}, \cdots, A_{2 n-3}\right) \\
& \quad=(-1)^{(n+1) / 2}\left((n-1) !\left(e_{11}+e_{22}\right)+2(n-2) !\left(e_{33}+\cdots+e_{n n}\right)\right)
\end{aligned}
$$

for $n$ odd.

Proof. First we shall prove by induction on $n$ that

$$
\begin{aligned}
S_{2 n-4}\left(A_{2}, \cdots, A_{2 n-3}\right) & \\
& =(-1)^{(n-2) / 2}\left((n-2) !\left(e_{11}+e_{22}\right)+2(n-3) !\left(e_{33}+\cdots+e_{n n}\right)\right)
\end{aligned}
$$

for $n$ even and

$$
S_{2 n-4}\left(A_{2}, \cdots, A_{2 n-3}\right)=(-1)^{(n+1 / 2}(n-2) !\left(e_{12}-e_{21}\right)
$$

for $n$ odd. This is trivial for $n=3, n=4$, so assume the assertion is true for all $m<n$. Let us evaluate

$$
\begin{aligned}
e_{11} S_{2 n-4} & \left(A_{2}, \ldots, A_{2 n-3}\right) \\
& =\sum_{i=2}^{2 n-3}(-1)^{i} e_{11} A_{i} S_{2 n-5}\left(A_{2}, \ldots, A_{i-1}, A_{i+1}, \ldots, A_{2 n+3}\right)
\end{aligned}
$$

by (1). Now $e_{11} A_{i}=0$ unless $i$ is even. Setting $i=2 r$ yields

$$
\begin{aligned}
(-1)^{2 r} e_{11} A_{2 r} S_{2 n-5}\left(A_{2}, \cdots, A_{2 r-1}, A_{2 r+1}, \cdots, A_{2 n-3}\right) \\
\quad=e_{1, r+2} S_{2 n-5}\left(A_{2}, \cdots, A_{2 r-1}, A_{2 r+1}, \cdots, A_{2 n-3}\right) \\
\quad=e_{1, r+2} A_{2 r+1} S_{2 n-6}\left(A_{2}, \cdots, A_{2 r-1}, A_{2 r+2}, \cdots, A_{2 n-3}\right) \\
\quad=e_{12} S_{2 n-6}\left(A_{2}, \cdots, A_{2 r-1}, A_{2 r+2}, \cdots, A_{2 n-3}\right) .
\end{aligned}
$$

Thus

$e_{11} S_{2 n-4}\left(A_{2}, \ldots, A_{2 n-3}\right)=\sum_{r=1}^{n-2} e_{12} S_{2 n-6}\left(A_{2}, \ldots, A_{2 r-1}, A_{2 r+2}, \ldots, A_{2 n-3}\right)$.

Now the index $r+2$ does not occur in $\left\{A_{2}, \cdots, A_{2 r-1}, A_{2 r+2}, \cdots, A_{2 n-3}\right\}$, so by induction 


$$
\begin{aligned}
& e_{12} S_{2(n-1)-4}\left(A_{2}, \cdots, A_{2 r-1}, A_{2 r+2}, \cdots, A_{2 n-3}\right) \\
& \quad=-(-1)^{n / 2}(n-3) ! e_{12} e_{21}=(-1)^{(n-2) / 2}(n-3) ! e_{11} \text { if } n \text { is even, } \\
& (-1)^{(n-3) / 2}(n-3) ! e_{12} e_{22}=(-1)^{(n+1) / 2}(n-3) ! e_{12} \text { if } n \text { is odd. }
\end{aligned}
$$

Hence $e_{11} S_{2 n-4}\left(A_{2}, \cdots, A_{2 n-3}\right)=(-1)^{(n-2) / 2}(n-2) ! e_{11}$ if $n$ is even, $(-1)^{(n+1) / 2}(n-2) ! e_{12}$ if $n$ is odd. The claim follows immediately from Lemmas 9 and 4.

Using this result we can now calculate $S_{2 n-3}\left(A_{1}, \cdots, A_{2 n-3}\right)$. Assume the given formula is valid for all $m<n$ (this is trivial for $n=2$ and $n=3$ ). As above,

$$
\begin{aligned}
e_{11} S_{2 n-3}\left(A_{1}, \cdots, A_{2 n-3}\right) & =e_{11} A_{1} S_{2 n-4}\left(A_{2}, \ldots, A_{2 n-3}\right) \\
& +\sum_{i=2}^{2 n-3}(-1)^{i-1} e_{11} A_{i} S_{2 n-4}\left(A_{1}, \ldots, A_{i-1}, A_{i+1}, \ldots, A_{2 n-3}\right) \\
= & e_{11} A_{1} S_{2 n-4}\left(A_{2}, \ldots, A_{2 n-3}\right) \\
& +\sum_{r=1}^{n-2} e_{12} S_{2 n-5}\left(A_{1}, \ldots, A_{2 r-1}, A_{2 r+2}, \ldots, A_{2 n-3}\right) .
\end{aligned}
$$

By induction (since the index $(r+2)$ does not occur in $\left\{A_{1}, \cdots, A_{2 r-1}, A_{2 r+2}\right.$, $\left.\left.\cdots, A_{2 n-3}\right\}\right)$,

$$
\begin{gathered}
e_{12} S_{2 n-5}\left(A_{1}, \cdots, A_{2 r-1}, A_{2 r+2}, \cdots, A_{2 n-3}\right) \\
=(-1)^{n / 2}(n-2) ! e_{12} \text { if } n \text { is even, } \\
-(-1)^{(n-1) / 2}(n-2) ! e_{12} e_{21}=(-1)^{(n+1) / 2}(n-2) ! e_{11} \text { if } n \text { is odd. }
\end{gathered}
$$

Thus $e_{11} S_{2 n-3}\left(A_{1}, \cdots, A_{2 n-3}\right)=(-1)^{n / 2}(n-1) ! e_{12}$ if $n$ is even, $(-1)^{(n+1) / 2}(n-1) ! e_{11}$ if $n$ is odd. The formula for $S_{2 n-3}\left(A_{1}, \ldots, A_{2 n-3}\right)$ is an immediate consequence of Lemmas 9 and 4. Q.E.D.

We shall now investigate the sharpness of Theorem 1 with the next three examples. The formula of Example 4 was observed first by Owens [5]. In these examples let $A_{2 r-1}=e_{r+1, r}-e_{r, r+1}$ and let $A_{2 r}=e_{r, r+2}-e_{r+2, r}, r \geq 1$.

Example 2. $s_{2 n-2}\left(e_{12}+e_{21}, A_{1}, \cdots, A_{2 n-3}\right)=0$ for $n$ odd, $2\left(e_{11}-e_{22}\right)$ for $n=2,4\left(e_{1, n-1}+e_{n-1,1}\right)$ for $n$ even $\geq 4$.

Proof. For $n$ odd, $S_{2 n-2}\left(e_{12}+e_{21}, A_{1}, \cdots, A_{2 n-3}\right)=0$ by $\mathcal{L}_{0}(n, 2 n-2,1)$, so we may assume $n$ is even. In this case $S_{2 n-2}\left(e_{12}+e_{21}, A_{1}, \cdots, A_{2 n-3}\right)$ is symmetric by Lemma 4. For $n=2, s_{2}\left(e_{12}+e_{21}, e_{21}-e_{12}\right)=2\left(e_{11}-e_{22}\right)$. For $n \geq 4$, the index 1 occurs in three matrices $\left(e_{12}+e_{21}, A_{1}\right.$, and $\left.A_{2}\right)$, so it suffices to show $e_{11} S_{2 n-2}\left(e_{12}+e_{21}, A_{1}, \ldots, A_{2 n-3}\right)=4 e_{1, n-1}$. Let $\Sigma_{1}$ be the sum of the 
monomials (of $e_{11} S_{2 n-2}\left(e_{12}+e_{21}, A_{1}, \ldots, A_{2 n-3}\right)$ ) in which $e_{12}+e_{21}$ and $A_{1}$ are adjacent, and let $\Sigma_{2}$ be the sum of the other monomials.

We claim $e_{11} s_{2 n-2}\left(e_{12}+e_{21}, A_{1}, \ldots, A_{2 n-3} ;\left(e_{12}+e_{21}\right) A_{1}\right)=0$. Indeed

$$
\begin{aligned}
e_{11} S_{2 n-2}\left(e_{12}\right. & \left.+e_{21}, A_{1}, \ldots, A_{2 n-3} ;\left(e_{12}+e_{21}\right) A_{1}\right) \\
& =e_{11}\left(e_{12}+e_{21}\right) A_{1} A_{2} S_{2 n-5}\left(A_{3}, \ldots, A_{2 n-3}\right) \\
& +e_{11} A_{2} S_{2 n-3}\left(\left(e_{12}+e_{21}\right), A_{1}, A_{3}, \ldots, A_{2 n-3} ;\left(e_{12}+e_{21}\right) A_{1}\right) \\
& =e_{13} S_{2 n-5}\left(A_{3}, \ldots, A_{2 n-3}\right) \\
& +e_{13} S_{2 n-3}\left(\left(e_{12}+e_{21}\right), A_{1}, A_{3}, \ldots, A_{2 n-3} ;\left(e_{12}+e_{21}\right) A_{1}\right)
\end{aligned}
$$

Consider a typical nonzero monomial $e_{13} A_{\pi 3} \cdots A_{\pi r}\left(e_{12}+e_{21}\right) A_{1} A_{\pi(r+1)} \cdots$ $A_{\pi(2 n-3)}$ where $\pi$ is a permutation of $(3, \ldots, 2 n-3)$. Since $\left(e_{12}+e_{21}\right) A_{1}=$ $e_{11}-e_{22}$ and since the index 1 does not occur in $\left\{A_{3}, \cdots, A_{2 n-3}\right\}$, it is clear that

$$
\begin{aligned}
e_{13} A_{\pi 3} & \cdots A_{\pi r}\left(e_{12}+e_{21}\right) A_{1} A_{\pi(r+1)} \cdots A_{\pi(2 n-3)} \\
& =e_{13} A_{\pi 3} \cdots A_{\pi r}\left(-e_{22}\right) A_{\pi(r+1)} \cdots A_{\pi(2 n-3)} \\
& =-e_{13} A_{\pi 3} \cdots A_{\pi r} A_{\pi(r+1)} \cdots A_{\pi(2 n-3)}
\end{aligned}
$$

Since 2 has degree 2 in $\Gamma\left(\left\{A_{3}, \ldots, A_{2 n-3}\right\}\right)$ we conclude that

$$
\begin{aligned}
e_{13} S_{2 n-3}\left(\left(e_{12}+e_{21}\right), A_{1}, A_{3}, \cdots, A_{2 n-3}\right. & \left.;\left(e_{12}+e_{21}\right) A_{1}\right) \\
& =-e_{13} S_{2 n-5}\left(A_{3}, \ldots, A_{2 n-3}\right)
\end{aligned}
$$

so

$$
e_{11} S_{2 n-2}\left(e_{12}+e_{21}, A_{1}, \cdots, A_{2 n-3} ;\left(e_{12}+e_{21}\right) A_{1}\right)=0 .
$$

Similarly,

$$
e_{11} S_{2 n-2}\left(e_{12}+e_{21}, A_{1}, \cdots, A_{2 n-3} ; A_{1}\left(e_{12}+e_{21}\right)\right)=0
$$

so $\Sigma_{1}=0$.

It suffices therefore to prove $\Sigma_{2}=4 e_{1, n-1}$ (for even $n \geq 4$ ). Since $\Sigma_{2}$ is the sum of those monomials in which $\left(e_{12}+e_{21}\right)$ and $A_{1}$ are nonadjacent, it is clear in view of the index 1 that no nonzero monomial of $\Sigma_{2}$ starts with $e_{11} A_{2}$; in fact, 


$$
\begin{aligned}
& \sum_{2}=e_{11}\left(e_{12}+e_{21}\right)\left(S_{2 n-3}\left(A_{1}, \ldots, A_{2 n-3} ; A_{2} A_{1} A_{3}\right)\right. \\
&\left.+S_{2 n-3}\left(A_{1}, \ldots, A_{2 n-3} ; A_{3} A_{1} A_{2}\right)\right) \\
&+ e_{11}\left(e_{12}+e_{21}\right)\left(S_{2 n-3}\left(A_{1}, \ldots, A_{2 n-3} ; A_{2} A_{1} A_{4}\right)\right. \\
&\left.+S_{2 n-3}\left(A_{1}, \cdots, A_{2 n-3} ; A_{4} A_{1} A_{2}\right)\right) \\
&-e_{11} A_{1}\left(S_{2 n-3}\left(\left(e_{12}+e_{21}\right), A_{2}, \cdots, A_{2 n-3} ; A_{2}\left(e_{12}+e_{21}\right) A_{3}\right)\right. \\
&\left.\quad+S_{2 n-3}\left(\left(e_{12}+e_{21}\right), A_{2}, \ldots, A_{2 n-3} ; A_{3}\left(e_{12}+e_{21}\right) A_{2}\right)\right) \\
&-e_{11} A_{1}\left(S_{2 n-3}\left(\left(e_{12}+e_{21}\right), A_{2}, \ldots, A_{2 n-3} ; A_{2}\left(e_{12}+e_{21}\right) A_{4}\right)\right. \\
&\left.\quad+S_{2 n-3}\left(\left(e_{12}+e_{21}\right), A_{2}, \ldots, A_{2 n-3} ; A_{4}\left(e_{12}+e_{21}\right) A_{2}\right)\right) .
\end{aligned}
$$

By Lemma 7 applied to each of the 4 pairs of terms, we see

$$
\begin{aligned}
\Sigma_{2} & =e_{12} S_{2 n-5}\left(2 e_{33}, A_{4}, \cdots, A_{2 n-3}\right)+e_{12} S_{2 n-5}\left(e_{34}+e_{43}, A_{3}, A_{5}, \cdots, A_{2 n-3}\right) \\
& +e_{12} S_{2 n-5}\left(0, A_{4}, \cdots, A_{2 n-3}\right)+e_{12}\left(S_{2 n-5}\left(-e_{34}+e_{43}, A_{3}, A_{5}, \cdots, A_{2 n-3}\right)\right.
\end{aligned}
$$

Clearly the last two terms are 0 since $S_{2 n-5}\left(0, A_{4}, \cdots, A_{2 n-3}\right)=0$ and

$$
\begin{aligned}
S_{2 n-5}\left(-e_{34}+\right. & \left.e_{43}, A_{3}, A_{5}, \ldots, A_{2 n-3}\right) \\
& =S_{2 n-5}\left(-e_{34}+e_{43}, A_{3}, e_{43}-e_{34}, \cdots, A_{2 n-3}\right)=0 .
\end{aligned}
$$

Thus

$$
\begin{aligned}
\sum_{2}=e_{12} S_{2 n-5} & \left(2 e_{33}, A_{4}, \ldots, A_{2 n-3}\right) \\
& +e_{12} S_{2 n-5}\left(e_{34}+e_{43}, A_{3}, A_{5}, \ldots, A_{2 n-3}\right) \\
= & -2 e_{12} A_{4} S_{2 n-6}\left(e_{33}, A_{5}, \ldots, A_{2 n-3}\right) \\
& -e_{12} A_{3} S_{2 n-6}\left(e_{33}+e_{43}, A_{5}, \ldots, A_{2 n-3}\right) \\
= & -2 e_{14} S_{2 n-6}\left(e_{33}, A_{5}, \ldots, A_{2 n-3}\right) \\
& +e_{13} S_{2 n-6}\left(e_{34}+e_{43}, A_{5}, \ldots, A_{2 n-3}\right)
\end{aligned}
$$

If $n=4$ then

$$
\Sigma_{2}=-2 e_{14} S_{2}\left(e_{33}, A_{5}\right)+e_{13} S_{2}\left(e_{34}+e_{43}, A_{5}\right)=+2 e_{13}+2 e_{13}=4 e_{13} .
$$

If $n>4$ then 


$$
\begin{aligned}
e_{14} S_{2 n-6} & \left(e_{33}, A_{5}, \ldots, A_{2 n-3}\right) \\
& =e_{14} S_{2 n-6}\left(e_{33}, A_{5}, \cdots, A_{2 n-3} ; A_{5} e_{33} A_{6}\right) \\
& +e_{14} S_{2 n-6}\left(e_{33}, \cdots, A_{5}, \cdots, A_{2 n-3} ; A_{6} e_{33} A_{5}\right) \\
& =e_{14} S_{2 n-8}\left(-e_{45}+e_{54}, A_{7}, \ldots, A_{2 n-3}\right)
\end{aligned}
$$

by Lemma 7. But $A_{7}=e_{54}-e_{45}$, so

$$
e_{14} S_{2 n-6}\left(e_{33}, A_{5}, \cdots, A_{2 n-3}\right)=e_{14} S_{2 n-8}\left(-e_{45}+e_{54}, e_{54}-e_{45}, \ldots\right)=0 \text {. }
$$

\section{Therefore}

$$
\Sigma_{2}=e_{13} s_{2 n-6}\left(e_{34}+e_{43}, A_{5}, \ldots, A_{2 n-3}\right)=e_{13}\left(4 e_{3, n-1}\right)=4 e_{1, n-1}
$$

by induction on $n$. Q.E.D.

Example 3. $S_{2 n-1}\left(e_{12}+e_{21}, A_{1}, \cdots, A_{2 n-3}, e_{n-1, n-1}\right)=4 e_{11}+2 e_{22}$ for $n=2$, $2 e_{12}-2 e_{21}$ for $n=3,4\left(e_{1, n-1}+e_{n-1,1}\right)$ for $n$ even $\geq 4,4\left(e_{n-1,1}-e_{1, n-1}\right)$ for $n$ odd $\geq 5$.

Proof. For $n=2, S_{3}\left(e_{12}+e_{21}, e_{21}-e_{12}, e_{11}\right)=4 e_{11}+2 e_{22}$. For $n>2$, the index 1 occurs in an odd number of matrices, as does the index $(n-1)$, so (by Lemma 4) $S_{2 n-1}\left(e_{12}+e_{21}, A_{1}, \ldots, A_{2 n-3}, e_{n-1, n-1}\right)=a_{n}\left(e_{1, n-1}+(-1)^{n} e_{n-1,1}\right), a_{n}$ in Z. We propose to show $a_{3}=+2, a_{n}=4$ for $n$ even $\geq 4$, and $a_{n}=-4$ for $n$ odd $\geq 5$.

Let $n=3$. Then $S_{5}\left(e_{12}+e_{21}, A_{1}, A_{2}, A_{3}, e_{22}\right) e_{22}=2 e_{12}$ is easy enough to see.

Let $n \geq 4$. Then

$$
\begin{aligned}
a_{n} e_{1, n-1}= & S_{2 n-1}\left(e_{12}+e_{21}, A_{1}, \ldots, A_{2 n-3}, e_{n-1, n-1}\right) e_{n-1, n-1} \\
= & S_{2 n-2}\left(e_{12}+e_{21}, A_{1}, \ldots, A_{2 n-3}\right) e_{n-1, n-1} \\
& -S_{2 n-2}\left(e_{12}+e_{21}, A_{1}, \ldots, A_{2 n-4}, e_{n-1, n-1}\right) A_{2 n-3} e_{n-1, n-1} \\
& -S_{2 n-2}\left(e_{12}+e_{21}, A_{1}, \ldots, A_{2 n-6}, A_{2 n-4}, A_{2 n-3}, e_{n-1, n-1}\right) A_{2 n-5} e_{n-1, n-1} \\
& +S_{2 n-2}\left(e_{12}+e_{21}, A_{1}, \ldots, A_{2 n-7}, A_{2 n-5}, \ldots, A_{2 n-3}, e_{n-1, n-1}\right) A_{2 n-6} e_{n-1, n-1} .
\end{aligned}
$$

Now, $S_{2 n-2}\left(e_{12}+e_{21}, A_{1}, \cdots, A_{2 n-3}\right) e_{n-1, n-1}=4 e_{1, n-1}$ if $n$ is even, 0 if $n$ is odd.

$$
\begin{aligned}
S_{2 n-2}\left(e_{12}+e_{21}, A_{1}, \cdots, A_{2 n-4}, e_{n-1, n-1}\right) e_{n, n-1} & \\
= & \left(S_{2 n-2}\left(e_{12}+e_{21}, A_{1}, \ldots, A_{2 n-4}, e_{n-1, n-1} ; A_{6} e_{n-1, n-1} A_{5}\right)\right. \\
& \left.\quad S_{2 n-2}\left(e_{12}+e_{21}, \ldots, A_{2 n-4}, e_{n-1, n-1} ; A_{5} e_{n-1, n-1} A_{6}\right)\right) e_{n, n-1} \\
& =-S_{2 n-4}\left(e_{12}+e_{21}, A_{1}, \ldots, A_{2 n-7}, e_{n-3, n-2}-e_{n-2, n-3}\right) e_{n, n-1}=0
\end{aligned}
$$


since $A_{2 n-7}=e_{n-3, n-2}-e_{n-2, n-3}$. Likewise,

$$
\begin{aligned}
& S_{2 n-2}\left(e_{12}+e_{21}, A_{1}, \cdots, A_{2 n-6}, A_{2 n-4}, A_{2 n-3}, e_{n-1, n-1}\right) e_{n-2, n-1} \\
& =\left(S_{2 n-2}\left(e_{12}+e_{21}, A_{1}, \ldots, A_{2 n-6}, A_{2 n-4}, A_{2 n-3}, e_{n-1, n-1} ; A_{2 n-6} e_{n-1, n-1} A_{2 n-3}\right)\right. \\
& \quad+S_{2 n-2}\left(e_{12}+e_{21}, A_{1}, \ldots, A_{2 n-6}, A_{2 n-4}, A_{2 n-3}, e_{n-1, n-1} ;\right. \\
& \left.\left.\quad A_{2 n-3} e_{n-1, n-1} A_{2 n-6}\right)\right) e_{n-2, n-1} \\
& =S_{2 n-4}\left(e_{12}+e_{21}, A_{1}, \ldots, A_{2 n-7},\left(e_{n-3, n}-e_{n, n-3}\right), A_{4}\right) e_{n-2, n-1} \\
& =-S_{2 n-4}\left(e_{12}+e_{21}, A_{1}, \ldots, A_{2 n-7}, e_{n-3, n}-e_{n, n-3}, e_{n, n-2}-e_{n-2, n}\right) e_{n-2, n-1}
\end{aligned}
$$

$=$ (by Example 2 for $(n-1)) 0$ if $n$ is even,

$$
=-4 e_{1, n-2} e_{n-2, n-1}=-4 e_{1, n-1} \text { if } n \text { is odd. }
$$

Finally,

$$
\begin{aligned}
S_{2 n-2}\left(e_{12}+e_{21}, A_{1}, \ldots, A_{2 n-7}, A_{2 n-5}, \cdots, A_{2 n-3}, e_{n-1, n-1}\right) e_{n-3, n-1} & \\
= & \left(S _ { 2 n - 2 } \left(e_{12}+e_{21}, A_{1}, \ldots, A_{2 n-7}, A_{2 n-5}, \cdots, A_{2 n-3}, e_{n-1, n-1} ;\right.\right. \\
& \left.A_{2 n-5} e_{n-1, n-1} A_{2 n-3}\right) \\
& +S_{2 n-2}\left(e_{12}+e_{21}, \ldots, A_{2 n-7}, A_{2 n-5}, \cdots, A_{2 n-3}, e_{n-1, n-1} ;\right. \\
& \left.\left.A_{2 n-3} e_{n-1, n-1}, A_{2 n-5}\right)\right) e_{n-3, n-1} \\
= & S_{2 n-4}\left(e_{12}+e_{21}, A_{1}, \ldots, A_{2 n-7},-e_{n-2, n}+e_{n, n-2}, A_{2 n-4}\right) e_{n-3, n-1}=0
\end{aligned}
$$

since $A_{2 n-4}=e_{n-2, n}-e_{n, n-2}$

Putting all of this together yields $a_{n} e_{1, n-1}=4 e_{1, n-1}+0+0+0=4 e_{1, n-1}$ for $n$ even $\geq 4 ; a_{n} e_{1, n-1}=0+0-4 e_{1, n-1}+0=-4 e_{1, n-1}$ for $n$ odd $\geq 5$, as desired. Q.E.D.

Example 4 (Owens [5]). $S_{2 n-3}\left(A_{1}, \cdots, A_{2 n-3}\right)=e_{21}-e_{12}$ for $n=2$, $2\left(e_{11}+e_{22}+e_{33}\right)$ for $n=3,2(n-1)\left(e_{2, n-1}-e_{n-1,2}\right)$ for $n$ even $\geq 4$, $2(n-2)\left(e_{2, n-1}-e_{n-1,2}\right)$ for $n$ odd $\geq 5$.

Proof. The cases $n=2$ and $n=3$ are immediate. For $n \geq 4$, it is clear from Lemma 4 that $S_{2 n-3}\left(A_{1}, \cdots, A_{2 n-3}\right)=a_{n}\left(e_{2, n-1}-(-1)^{n} e_{n-1,2}\right)$ for suitable $a_{n}$ in Z. We shall show $a_{n}=2(n-1)$ for $n$ even, $2(n-2)$ for $n$ odd.

$$
\begin{aligned}
a_{n} e_{2, n-1}= & e_{22} S_{2 n-3}\left(A_{1}, \cdots, A_{2 n-3}\right) \\
= & e_{22} A_{1} S_{2 n-4}\left(A_{2}, \cdots, A_{2 n-3}\right)+e_{22} A_{3} S_{2 n 4}\left(A_{1}, A_{2}, A_{4}, \ldots, A_{2 n-3}\right) \\
& -e_{22} A_{4} S_{2 n-4}\left(A_{1}, A_{2}, A_{3}, A_{3}, \cdots, A_{2 n-3}\right) .
\end{aligned}
$$




$$
\begin{aligned}
e_{21} S_{2 n-4}\left(A_{2}, \ldots, A_{2 n-3}\right) & =e_{21} A_{2} S_{2 n-5}\left(A_{3}, \ldots, A_{2 n-3}\right) \\
& =e_{23} a_{n-1} e_{3, n-1}=a_{n-1} e_{2, n-1} \\
e_{23} S_{2 n-4}\left(A_{1}, A_{2}, A_{4}, \ldots, A_{2 n-3}\right) & \\
= & e_{23}\left(S_{2 n-4}\left(A_{1}, A_{2}, A_{4}, \ldots, A_{2 n-3} ; A_{2} A_{1} A_{4}\right)\right. \\
& \left.+S_{2 n-4}\left(A_{1}, A_{2}, A_{4}, \ldots, A_{2 n-3} ; A_{4} A_{1} A_{2}\right)\right) \\
= & e_{23} S_{2 n-6}\left(e_{34}+e_{43}, A_{5}, \ldots, A_{2 n-3}\right)=0
\end{aligned}
$$

for $n$ odd, $e_{23}\left(2 e_{33}\right)=2 e_{23}$ for $n=4, e_{23}\left(4 e_{3, n-1}\right)=4 e_{2, n-1}$ for $n$ even $>4$, by Example 1 applied to $(n-2)$. Finally, $e_{24} S_{2 n-4}\left(A_{1}, A_{2}, A_{3}, A_{5}, \cdots, A_{2 n-3}\right)=$ $e_{24} S_{2 n-6}\left(2 e_{33}, A_{5}, \ldots, A_{2 n-3}\right)=-2 e_{23}$ for $n=4$; otherwise $2 e_{24} S_{2 n-6}\left(e_{33}, A_{5}, \cdots, A_{2 n-3}\right)=2 e_{24} S_{2 n-8}\left(e_{54}-e_{45}, A_{7}, \cdots, A_{2 n-3}\right)=0$ since $A_{7}=$ $e_{54}-e_{45}$.

Putting everything together, we have $a_{4}=a_{3}+2-(-2)=6$; for $n$ even $>4$ (proceeding inductively) $a_{n}=a_{n-1}+4+0=2((n-1)-2)+4=2(n-1)$; for $n$ odd (proceeding inductively) $a_{n}=a_{n-1}+0+0=2((n-1)-1)=2(n-2)$. Q.E.D.

We are prepared now to examine $\mathscr{L}_{p}(n, k, t)$ for $p \neq 2$. First let $t=0$. We have proved $\mathscr{L}_{p}(n, k, 0)$ for $k \geq 2 n-2$. Example 4 shows $\mathscr{L}_{p}(n, 2 n-3,0)$ is false for $p \nmid 2[n / 2]-1$. Lemma $1(b)$ then shows $\mathscr{Q}_{p}(n, 2 n-4,0)$ is false for $p \gamma 2[n / 2]-1$. In fact, if $n$ is even then $\mathscr{Q}_{p}(n, 2 n-4,0)$ is false for all $p \neq 2$. For suppose $\mathscr{L}_{p}(n, 2 n-4,0)$. Then $p \mid 2[n / 2]-1=n-1$. But Lemma 2 would imply $\mathscr{L}_{p}(n-1,2(n-1)-3,0)$, so $p \mid 2[(n-1) / 2]-1=n-3$. Thus $p \mid((n-1)-(n-3))=2$, so $p=2$. Since $\mathscr{E}_{p}(n, 2 n-4,0)$ is false for $n$ even, all $p \neq 2$, we conclude by Lemmas $1(b)$ and 2 that $\mathcal{L}_{p}(n, k, 0)$ is false for all $p \neq 2$, all $n, k \leq 2 n-4$. Thus, for $n$ even we have determined the truth or falsehood of $\mathscr{L}_{p}(n, k, 0)$ in all cases except $n \geq 8, k=2 n-3, p \mid n-1$. For $n$ odd we have determined the truth or falsehood of $\mathfrak{L}_{p}(n, k, 0)$ in all cases except $n \geq 9, k=$ $2 n-3$ or $k=2 n-4, p \mid n-2$.

Next let $t=1$. We have proved $\mathcal{L}_{p}(n, k, 1)$ for $k \geq 2 n-1$ and $\mathcal{L}_{p}(n, 2 n-2,1)$ for $n$ odd. If $p \neq 2$ then Example 2 shows $\mathcal{Q}_{p}(n, 2 n-2,1)$ is false for $n$ even, and it follows by Lemma 2 that $\mathscr{L}_{p}(n, 2 n-3,1)$ is false for $n$ odd, all $p \neq 2$. We claim that $\mathcal{L}_{p}(n, 2 n-3,1)$ is also false for $n$ even, all $p \neq 2$. Indeed, suppose $\mathcal{L}_{p}(n, 2 n-3,1)$. Using the notation of Example 2, we clearly have (for $n \geq 4$ )

$$
\begin{aligned}
4 e_{1, n-1}= & S_{2 n-2}\left(e_{12}+e_{21}, A_{1}, \ldots, A_{2 n-3}\right) e_{n-1, n-1} \\
= & S_{2 n-3}\left(e_{12}+e_{21}, A_{1}, \ldots, A_{2 n-4}\right) A_{2 n-3} e_{n-1, n-1} \\
& \quad+S_{2 n-3}\left(e_{12}+e_{21}, A_{1}, \ldots, A_{2 n-6}, A_{2 n-4}, A_{2 n-3}\right) A_{2 n-5} e_{n-1, n-1} \\
& \quad-S_{2 n-3}\left(e_{12}+e_{21}, A_{1}, \ldots, A_{2 n-7}, A_{2 n-5}, \ldots, A_{2 n-3}\right) A_{2 n-6} e_{n-1, n-1}
\end{aligned}
$$


Now each of the right-hand terms would be 0 by $\mathfrak{L}_{p}(n, 2 n-3,1)$, which would imply $4 \equiv 0(\bmod p)$. This is impossible unless $p=2$, thereby establishing the claim. It follows through fepeated applications of Lemma 2 that $\mathscr{L}_{p}(n, k, 1)$ is false for all $n$, all $p \neq 2, k \leq 2 n-3$. Hence for $t=1$ the statement of Theorem 1 is sharp for ali characteristics $\neq 2$.

Finally, let $t \geq 2$. Example 3 shows $\mathfrak{Q}_{p}(n, 2 n-1,2)$ is false for all $n, p \neq 2$, and applications of Lemma 2 then show $\mathfrak{L}_{p}(n, k, 2)$ is false for all $n, p \neq 2, k \leq$ $2 n-1$. We conclude that $\mathfrak{L}_{p}(n, k, t)$ is false for all $n, p \neq 2, t \geq 2, k \leq 2 n-1$, from the following easy remark:

Remark. Let $A_{1}, \cdots, A_{k} \in M_{n}(F)$. Then $S_{k+2}\left(A_{1}, \cdots, A_{k}, e_{i, n+1}-e_{n+1, i}\right.$, $\left.e_{n+1, n+1}\right)=S_{k}\left(A_{1}, \cdots, A_{k}\right) e_{i, n+1}+e_{n+1, i} S_{k}\left(A_{1}, \cdots, A_{k}\right)$.

Summarizing the above counterexamples, we see that the following sentences are false:

$\mathcal{L}_{p}(n, k, 0)$ for all $n, p \neq 2, k \leq 2 n-5$.

$\mathcal{L}_{p}(n, 2 n-4,0)$ for all even $n, p \neq 2$.

$\mathfrak{L}_{p}(n, 2 n-3,0), \mathfrak{L}_{p}(n+1,2(n+1)-3,0), \mathfrak{L}_{p}(n+1,2(n+1)-4,0)$ for $p \nmid 2(n-1), n$ even. (These three sentences are equivalent for any $p$.

$\mathscr{L}_{p}(n, k, 1)$ for all $n, p \neq 2, k \leq 2 n-3$.

$\mathcal{L}_{p}(n, 2 n-2,1)$ for all even $n, p \neq 2$.

$\mathfrak{L}_{p}(n, k, t)$ for all $n, p \neq 2, k \leq 2 n-1, t \geq 2$.

In particular, Theorem 1 is sharp for characteristic 0 and for $t \geq 1$ in arbitrary characteristic $\neq 2$.

If $p=2$ the following example shows that Theorem 2 is as sharp as possible:

Example 5. Let $A_{i}=e_{i, i+1}-e_{i+1, i}, 1 \leq i \leq n-1$. Then for any $t \leq n$, $S_{n+t-1}\left(A_{1}, \cdots, A_{n-1}, e_{11}, \cdots, e_{t t}\right)=e_{1 n} \pm \cdot e_{n 1}$.

Proof. Immediate.

9. Identities in matrix algebras with involution. Let $\Omega$ be any domain and let (*) be the transpose on $M_{n}(\Omega)$ with respect to a suitable set of matric units $\left\{e_{i j} \mid 1 \leq i \leq j \leq n\right\}$. Clearly $(*)$ is an antiautomorphism of degree 2 , otherwise called an involution, and we let $\left.M_{n}(\Omega), *\right)$ denote this matrix algebra with involution.

Now let $\Omega\{X\}=\Omega\left\{X_{11}, X_{12}, \cdots, X_{i 1}, X_{i 2}, \ldots\right\}$ be the free noncommutative $\Omega$-algebra generated over $\Omega$ by the countable set of noncommutative indeterminates $\left\{X_{11}, X_{12}, \cdots, X_{i 1}, X_{i 2}, \cdots\right\} . \Omega\{X\}$ can be given the involution $(*)$ defined by $\omega^{*}=\omega$, all $\omega$ in $\Omega$, and $X_{i 1}^{*}=X_{i 2}, X_{i 2}^{*}=X_{i 1}$, all $i$. Let us write $X_{i}=X_{i 1}$, $X_{i}^{*}=X_{i 2}$. A homomorphism of $(\Omega\{X\}, *)$ to $\left(M_{n}(\Omega), *\right)$ is a homomorphism of $\Omega\{X\}$ to $M_{n}(\Omega)$ which preserves the involution. Suppose a nonzero element 
$f\left(X_{1}, X_{1}^{*}, \cdots, X_{m}, X_{m}^{*}\right)$ of $(\Omega\{X\}, *)$ is in the kernel of every homomorphism from $(\Omega\{X\}, *)$ to $\left(M_{n}(\Omega), *\right)$. Then we say $f$ is an identity of $\left(M_{n}(\Omega), *\right)$. Identities in matrix algebras with involution have been a source of interest in recent years, and we shall see in this section how the results of this paper tie in with the theory of identities of $\left(M_{n}(\Omega), *\right)$ for arbitrary $\Omega$.

Viewing $f$ as a sum of monomials $f_{r}\left(X_{1}, X_{1}^{*}, \cdots, X_{m}, X_{m}^{*}\right)$, we shall say the degree of the ith indeterminate of $f_{r}$ is the sum of the degrees of $X_{i}$ and of $X_{i}^{*}$ in $f_{r}$. The degree of $f_{r}$ is the sum of the degrees of the indeterminates of $f_{r}$, and the degree of $f$ is the maximal degree of its monomials. The polynomial $f$ is multilinear if each indeterminate occurring nontrivially in $f$ has degree 1 in each monomial of $f$. There is a well-known procedure, given an identity $f$ of $\left(M_{n}(\Omega), *\right)$, to obtain a multilinear identity $\hat{f}$ of $\left(M_{n}(\Omega), *\right)$, with degree not greater than that of $f$.

Now let $g\left(Y_{1}, \cdots, Y_{k}\right)$ be a polynomial with coefficients in $\Omega$. If $g\left(A_{1}, \cdots, A_{k}\right)=0$ for all possible $A_{1}, \cdots, A_{k-t}$ in $M_{n}^{-}(\Omega)$ and $A_{k-t+1}, \cdots, A_{k}$ in $M_{n}^{+}(\Omega)$ we shall say $g$ is a $(k-t, t)$-identity of $M_{n}(\Omega)$ (with respect to $(*)$ ).

Theorems 1 and 2 and Proposition 2, Corollaries 1 and 2 provide various standard $(k-t, t)$-identities of $M_{n}(\Omega)$.

Suppose char $\Omega \neq 2$. Then any $(k-t, t)$-identity $g\left(Y_{1}, \ldots, Y_{k}\right)$ yields the identity $g\left(X_{1}-X_{1}^{*}, \ldots, X_{k-t}-X_{k-t}^{*}, X_{k-t+1}+X_{k-t+1}^{*}, \cdots, X_{k}+X_{k}^{*}\right)$ of $\left(M_{n}(\Omega), *\right)$, since $X-X^{*}$ is antisymmetric in $\Omega\{X\}$ and $X+X^{*}$ is symmetric in $\boldsymbol{\Omega}\{X\}$. If $g\left(Y_{1}, \cdots, Y_{k}\right)$ is multilinear then so is $g\left(X_{1}-X_{1}^{*}, \cdots, X_{k-t}-X_{k-t}^{*}\right.$, $\left.X_{k-t+1}+X_{k-t+1}^{*}, \cdots, X_{k}+X_{k}^{*}\right)$. Conversely, let $f\left(X_{1}, X_{1}^{*}, \cdots, X_{m}, X_{m}^{*}\right)$ be a multilinear identity of $\left(M_{n}(\Omega), *\right)$. Then $f\left(x_{1}, x_{1}^{*}, \cdots, x_{m}, x_{m}^{*}\right)=$ $f_{1}\left(x_{1}, x_{2}, x_{2}^{*}, \cdots, x_{m}, x_{m}^{*}\right)+f_{2}\left(x_{1}^{*}, x_{2}, x_{2}^{*}, \ldots, x_{m}, x_{m}^{*}\right)$. Evidently $f_{1}\left(x_{1}^{*}, x_{2}^{*}, \cdots, x_{m}, x_{m}^{*}\right)+f_{2}\left(x_{1}, x_{2}, x_{2}^{*}, \cdots, x_{m}, x_{m}^{*}\right)$ is also an identity $\hat{f}$ of $\left(M_{n}(\Omega), *\right)$. If $\hat{f}=f$ then $f\left(X_{1}, X_{1}^{*}, \cdots, x_{m}, x_{m}^{*}\right)=f_{1}\left(X_{1}+x_{1}^{*}, X_{2}, x_{2}^{*}, \cdots, X_{m}, x_{m}^{*}\right)$. Otherwise $f-\hat{f}=f_{1}\left(X_{1}-X_{1}^{*}, X_{2}, X_{2}^{*}, \ldots, X_{m}, X_{m}^{*}\right)-f_{2}\left(X_{1}-X_{1}^{*}, X_{2}, X_{2}^{*}, \cdots, X_{m}, X_{m}^{*}\right)$ which is also an identity of $\left(M_{n}(\Omega), *\right)$. Continuing in this manner yields a multilinear identity $g\left(X_{1}-X_{1}^{*}, \cdots, X_{m-t}-X_{m-t}^{*}, X_{m-t+1}+X_{m-t+1}^{*}, \cdots, X_{m}+X_{m}^{*}\right)$ for suitable $t$ so $g\left(Y_{1}, \cdots, Y_{m}\right)$ is a $(m-t, t)$-identity of $M_{n}(\Omega)$ (with respect to $(*))$.

Thus we have a way of passing back and forth between multilinear identities of matrix algebras with transpose and polynomials of the type investigated in this paper. In particular, $S_{2 n-2}\left(X_{1}-X_{1}^{*}, \cdots, X_{2 n-2}-X_{2 n-2}^{*}\right)$ is an identity in $\left(M_{n}(\Omega), *\right),(*)$ the transpose involution, for domains $\Omega$ of characteristic $\neq 2$. On the other hand, $S_{2 n-2}\left(X_{11}-X_{12}, \cdots, X_{2 n-2,1}-X_{2 n-2,2}\right)$ obviously is not an 
identity of $M_{n}(\Omega)$ in the usual sense, so we have obtained identities of $\left(M_{n}(\Omega), *\right)$ which are not consequences of the identities of $M_{n}(\Omega)$. Viewed in this light, this paper becomes a study of standard identities of $\left(M_{n}(\Omega), *\right)$ which are not consequences of identities of $M_{n}(\Omega)$. Applications of this study and its analogous results when $(*)$ is an arbitrary involution may be found in [6].

\section{REFERENCES}

1. S. A. Amitsur and J. Levitzki, Minimal identities for algebras, Proc. Amer. Math. Soc. 1 (1950), 449-463. MR 12, 155.

2. N. Jacobson, Structure of rings, 2nd rev. ed., Amer. Math. Soc. Colloq. Publ., vol. 37, Amer. Math. Soc., Providence, R.I., 1964, Chap. X. MR 36 \#158.

3. B. Kostant, $A$ theorem of Frobenius, a theorem of Amitsur-Levitzki, and cohomology theory, J. Math. Mech. 7 (1958), 237-264. MR 19, 1153.

4. Oystein Ore, Theory of graphs, Amer. Math. Soc. Colloq. Publ., vol. 38, Amer. Math. Soc., Providence, R.I., 1962. MR 27 \#740.

5. F. Owens, Applications of graph theory to matrix theory (to appear).

6. L. Rowen, Thesis, Yale University, New Haven, Conn., 1973.

DEPARTMENT OF MATHEMATICS, YALE UNIVERSITY, NET HAVEN, CONNECTICUT 06520 60637

Current address: Department of Mathematics, University of Chicago, Chicago, Illinois 